\title{
Characterizing Optical Fiber Transmission Matrices Using Metasurface Reflector Stacks for Lensless Imaging without Distal Access
}

\author{
George S. D. Gordon $\odot,{ }^{1, *}$ Milana Gataric, ${ }^{2}$ Alberto Gil C. P. Ramos, ${ }^{3}$ Ralf Mouthaan, ${ }^{1,4}$ Calum Williams, ${ }^{1}$ \\ Jonghee Yoon, ${ }^{4,5}$ Timothy D. Wilkinson, ${ }^{1}$ and Sarah E. Bohndiek ${ }^{4,5}$ \\ ${ }^{1}$ Electrical Engineering Division, Department of Engineering, University of Cambridge, 9 J.J. Thomson \\ Avenue, Cambridge CB3 OFA, United Kingdom \\ ${ }^{2}$ Department of Pure Mathematics and Mathematical Statistics, Centre for Mathematical Sciences, \\ Wilberforce Road, Cambridge CB3 OWB, United Kingdom \\ ${ }^{3}$ Nokia, Broers Building, 21 J.J. Thomson Avenue, Cambridge CB3 OFA, United Kingdom \\ ${ }^{4}$ Department of Physics, Cavendish Laboratory, 19 J.J. Thomson Avenue, \\ Cambridge CB3 OHE, United Kingdom \\ ${ }^{5}$ CRUK Cambridge Institute, University of Cambridge, Li Ka Shing Centre, Robinson Way, \\ Cambridge CB2 ORE, United Kingdom
}

(Received 30 April 2019; revised manuscript received 29 August 2019; published 9 December 2019)

\begin{abstract}
The ability to retrieve image data through hair-thin optical fibers promises to open up new applications in a range of fields, from biomedical imaging to industrial inspection. Unfortunately, small changes in mechanical deformation and temperature can completely scramble optical information, distorting any resulting images. Correction of these dynamic changes requires measurement of the fiber transmission matrix (TM) in situ immediately before imaging, which typically requires access to both the proximal and distal facets of the fiber simultaneously. As a result, TM calibration is not feasible during most realistic usage scenarios without compromising the thin form factor with bulky distal optics. Here, we introduce a new approach to determine the TM of multimode or multicore optical fibers in a reflection-mode configuration, without requiring access to the distal facet. We propose introducing a thin stack of structured metasurface reflectors at the distal facet of the fiber, to introduce wavelength-dependent, spatially heterogeneous reflectance profiles. We derive a first-order fiber model that compensates these wavelength-dependent changes in the fiber TM and show that, consequently, the reflected data at three wavelengths can be used to unambiguously reconstruct the full TM by an iterative optimization algorithm. Unlike previous approaches, our method does not require the fiber matrix to be unitary, making it applicable to physically realistic fiber systems that have non-negligible power loss. We demonstrate TM reconstruction and imaging first using simulated nonunitary fibers and noisy reflection matrices, then using larger experimentally measured TMs of a densely packed multicore fiber (MCF), and finally using experimentally measured multiwavelength TMs recorded from a step-index multimode fiber (MMF). Parallelization of multiwavelength in situ measurements could enable experimental characterization times comparable with state-of-the-art transmission-mode fiber TM experiments. Our findings pave the way for online TM calibration in situ in hair-thin optical fibers.

DOI: 10.1103/PhysRevX.9.041050

Subject Areas: Metamaterials, Optics
\end{abstract}

\section{INTRODUCTION}

Lensless imaging through hair-thin optical fibers is a technique that promises to open up new areas in biomedical imaging such as the following: in vivo

\footnotetext{
*Present address: Department of Electrical and Electronic Engineering, The University of Nottingham, University Park, Nottingham NG7 2RD, United Kingdom.

Published by the American Physical Society under the terms of the Creative Commons Attribution 4.0 International license. Further distribution of this work must maintain attribution to the author(s) and the published article's title, journal citation, and DOI.
}

bright-field, dark-field, and fluorescence microscopy deep in living brains [1,2]; quantitative phase and polarimetric imaging with applications in early detection of cancer $[3,4]$; and endoscopic confocal microscopy for high-resolution imaging [5]. For all these applications, accurate characterization of the deterministic propagation of light through the fiber, discretized as the transmission matrix (TM) [6], is essential for imaging, whether used directly [4], or indirectly via phase conjugation [7] or via optimization [8].

Unfortunately, the TM is highly sensitive to small changes in mechanical deformation and temperature that are unavoidable in living subjects, so the TM should be calibrated immediately before imaging. The most direct way of measuring a TM is to send predefined optical fields 
into the distal facet of the fiber (furthest from the operator) and measure the resulting fields at the proximal facet (nearest the operator). This widely-used approach has a critical limitation: The distal facet of the fiber is deep within the subject during imaging, so the predefined optical fields cannot be reliably generated without additional bulky distal optics that would compromise the ultrathin form factor.

Several methods have been proposed to overcome this limitation. Placing a holographic plate on the distal facet of the fiber, illuminated via a separate single-mode fiber, creates a "virtual beacon" [9] that enables partial recovery of the TM, sufficient for limited confocal imaging but not wide-field imaging (e.g., quantitative phase). Highly accurate modeling of TM perturbations is an effective approach when the fiber refractive index and bending configuration are known precisely, but it performs best for relatively short lengths of fiber (less than $10 \mathrm{~cm}$ ) [10]. Distal reflector assemblies that introduce characteristic time delays have been demonstrated but require a unique reflector for each characterized propagation mode, which does not scale well for high-resolution imaging applications [11].

$\mathrm{Gu}$ et al. proposed a method that does not require prior knowledge of the refractive index and infers the forward TM based on the reflection matrix (RM), i.e., light that has made a round trip into the proximal facet, off a distal reflector, and back out the proximal facet [12]. The transpose symmetry of fiber TMs inherently means that such RMs exhibit a quadratic relationship with their respective TMs, resulting in ambiguities during recovery $[12,13]$. These ambiguities reduce to an $N$-dimensional vector in $\{-1,1\}^{N}$ (for an $N$-pixel image) when using a multimode fiber (MMF) that is near perfectly lossless (unitary) [12] and can then be assumed as invariants of the fiber and measured in advance [12]. There are two key shortcomings of this approach: First, in practice, even precision-manufactured MMF TMs are nonunitary $[14,15]$ because high-order modes, with more power concentrated in the fiber cladding, tend to exhibit power leakage at bends [16]. Second, light must be able to pass through the distal end to enable imaging but should not couple back in during RM measurement. $\mathrm{Gu}$ et al. addressed this by proposing to include a shutter at the distal facet, but again, this added bulk would compromise the ultrathin form factor [12].

Seeking an approach that is agnostic to the fiber refractive index but maintains the hair-thin property of the fibers, we demonstrate here a new method that enables measurement of nonunitary fiber TMs of arbitrary size without access to the distal facet and without adding distal bulk, making it suitable for ultrathin flexible imaging devices. The key innovation is a distal reflector, comprising a multilayer stack of spatially heterogeneous, partially reflecting, wire-grid polarizers (termed "metasurfaces") and long-pass optical filters, that is feasible to fabricate by combining previously validated approaches [17-19]. The filter stack creates a spatially and spectrally varying reflector, whose properties can be characterized prior to use and will remain fixed throughout any imaging experiment. We show that by combining multiple RMs recorded at different wavelengths, it is possible to estimate the instantaneous TM and then perform imaging at another wavelength.

Using a first-order multiwavelength fiber model, we first introduce a novel iterative optimization method that infers the TM based on three RMs measured at different wavelengths and prior knowledge of the reflector properties. This method does not require the TM to be unitary, only that it is invertible, making it applicable to lossy fibers or general scattering media. Following TM reconstruction, we then show how imaging can be performed at a fourth wavelength. Next, we computationally demonstrate the method using simulated $32 \times 32$ nonunitary fiber matrices with noise. We then demonstrate the recovery algorithm using an experimentally measured multicore fiber (MCF) TM $(1648 \times 1648)$ and a realistically simulated reflector stack, showing that the method can be scaled up effectively. Finally, we demonstrate TM recovery using an experimentally measured multiwavelength TM of a step-index MMF validating both that the method works for real MMF and that the first-order physical model used to predict changes in TM with wavelength is physically realistic. Our findings underpin a compelling new approach for enabling ultrathin flexible lensless imaging via optical fibers.

\section{THEORY}

\section{A. Physical model for TM characterization in reflection mode}

The physical model used for reference in this paper is shown in Fig. 1. We consider a well-established model where an optical field containing $M$ pixels, each a complexvalued vector encoding amplitude and phase in two orthogonal polarizations, is recorded. The sampled field

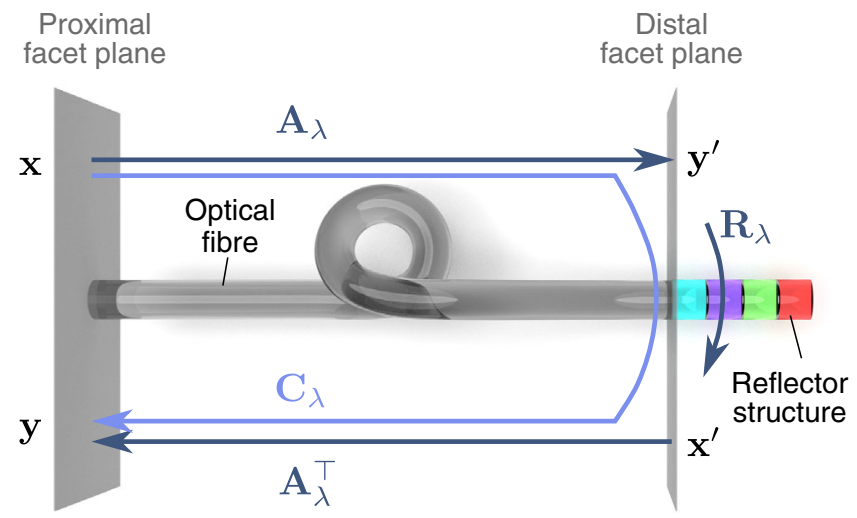

FIG. 1. Physical model used for fiber TM characterization in reflection mode. The proximal facet of the fiber is nearest to the imaging system (i.e., outside of the subject), while the distal facet is nearest the object being imaged (i.e., inside the subject). Vectors at the distal facet are denoted using the prime symbol ('). Here, $\mathbf{A}_{\lambda}$ represents the fiber TM at wavelength $\lambda, \mathbf{R}_{\lambda}$ the PM of the reflector stack, and $\mathbf{C}_{\lambda}$ the round-trip $\mathrm{RM}$, i.e., down the fiber, off the reflector, back up the fiber. 
at some input plane (e.g., the proximal facet) is ordered into a vector $\mathbf{x} \in \mathbb{C}^{2 M}$, and the sampled field at an output plane (e.g., the distal facet) is similarly ordered into a vector $\mathbf{y}^{\prime} \in \mathbb{C}^{2 M}$. In the forward propagation direction, these vectors are related by the monochromatic fiber TM at some wavelength, $\lambda, \mathbf{A}_{\lambda} \in \mathbb{C}^{2 M \times 2 M}[6,20]$ :

$$
\mathbf{y}^{\prime}=\mathbf{A}_{\lambda} \mathbf{x} .
$$

Similarly, if we consider a field, $\mathbf{x}^{\prime} \in \mathbb{C}^{2 M}$, at the distal facet propagating in the reverse direction to become a field, $\mathbf{y} \in \mathbb{C}^{2 M}$, at the proximal facet, these are related by the transpose of the fiber TM [12]:

$$
\mathbf{y}=\mathbf{A}_{\lambda}^{\top} \mathbf{x}^{\prime} .
$$

We then consider the impact of adding a reflector at the distal facet of the fiber. In general, the reflector is considered to be spatially heterogeneous in terms of its localized Jones reflection matrices: There may be uncorrelated Jones matrices describing reflections at each spatial point. Furthermore, if the reflector is offset from the fiber, light may couple between spatial positions due to diffraction. This behavior is linear, so it is represented by a partial reflector matrix (PM) at wavelength $\lambda, \mathbf{R}_{\lambda} \in \mathbb{C}^{2 M \times 2 M}$, that relates $\mathbf{y}^{\prime}$ and $\mathbf{x}^{\prime}$ (Fig. 1):

$$
\mathbf{x}^{\prime}=\mathbf{R}_{\lambda} \mathbf{y}^{\prime} .
$$

Next, combining Eqs. (1)-(3), we can determine the optical field exiting the proximal facet in the reverse direction:

$$
\mathbf{y}=\mathbf{A}_{\lambda}^{\top} \mathbf{R}_{\lambda} \mathbf{A}_{\lambda} \mathbf{x}=\mathbf{C}_{\lambda} \mathbf{x},
$$

where

$$
\mathbf{C}_{\lambda}=\mathbf{A}_{\lambda}^{\top} \mathbf{R}_{\lambda} \mathbf{A}_{\lambda} \in \mathbb{C}^{2 M \times 2 M}
$$

is a RM. Physically speaking, $\mathbf{C}_{\lambda}$ represents light taking a complete round trip (or double pass): down the fiber, off a given reflector, and back up the fiber (Fig. 1). Experimentally, it is determined through multiple measurements of vector pairs, $(\mathbf{x}, \mathbf{y})$, at the proximal facet of the fiber. This process may lead to a nonsquare approximation to $\mathbf{C}_{\lambda}$, denoted as $\tilde{\mathbf{C}}_{\lambda}$. To enable computation of matrix exponentials, $\tilde{\mathbf{C}}_{\lambda}$ first needs to be downsampled into a square matrix $\tilde{\tilde{\mathbf{C}}}_{\lambda}$, which is then used as a surrogate for $\mathbf{C}_{\lambda}$ in what follows (Appendix A 1).

Given that we can characterize $\mathbf{R}_{\lambda}$ in advance (Appendix A 2), and it will remain fixed throughout its use, the goal is then to recover $\mathbf{A}_{\lambda}$ based on measurements of $\mathbf{C}_{\lambda}$ and $\mathbf{R}_{\lambda}$. At a single wavelength $\lambda$, this goal is not possible, in general, unless the fiber TM is unitary $[12,14]$. We therefore propose to use several different reflectors with PMs $\mathbf{R}_{\lambda}$ at wavelengths $\lambda=\lambda_{1}, \ldots, \lambda_{Q}$, and measured RMs $\mathbf{C}_{\lambda}, \lambda=\lambda_{1}, \ldots, \lambda_{Q}$, to enable unambiguous recovery of $\mathbf{A}_{\lambda}$ for any $\lambda=\lambda_{1}, \ldots, \lambda_{Q}$ in the more general nonunitary case.

In order to recover $\mathbf{A}_{\lambda}$ for any $\lambda=\lambda_{1}, \ldots, \lambda_{Q}$, given $R_{\lambda_{q}}$, $C_{\lambda_{q}}$ with $q=1, \ldots, Q$ and Eq. (5), we must model the relationship between TMs at different wavelengths. We consider, in turn, two simple models: a "zeroth-order model" and a "first-order model".

\section{B. Zeroth-order model and TM recovery}

The zeroth-order model assumes that the TMs under the different reflectors are the same, i.e.,

$$
\mathbf{A}=\mathbf{A}_{\lambda_{1}}=\mathbf{A}_{\lambda_{2}}=\ldots=\mathbf{A}_{\lambda_{Q}} .
$$

Physically, this corresponds to wavelength modulations significantly less than the spectral bandwidth of the fiber (see Appendix B). This model has the significant advantage that if at least three wavelengths are used $(Q \geq 3)$ producing at least three RMs, $\mathbf{A}$ can be recovered in a relatively straightforward way, relying largely on analytical steps (Appendix C). This analytical approach further requires that the eigenvalues of each $\mathrm{PM}, \mathbf{R}_{\lambda}, \lambda=\lambda_{1}, \ldots, \lambda_{Q}$, must be distinct for unambiguous TM recovery, in agreement with previous findings [12]. Light must therefore be coupled between different fiber modes and polarizations, so a conventional partial mirror reflector would not work because it preserves polarization states leading to repeated matrix eigenvalues. This insight informs reflector design. In reality, the relatively small spectral bandwidths of typical imaging fibers (about $5 \mathrm{~nm}$ ) would require the use of optical components (e.g., filters) with impractically sharp spectral responses to achieve different reflectance behavior over such a small wavelength range.

\section{First-order model and TM recovery}

Given the limitations of the zeroth-order approach, we next consider a model that relates TMs at different wavelengths, $\lambda_{p}$ and $\lambda_{q}$, as

$$
\mathbf{A}_{\lambda_{q}}=e^{\left(\lambda_{p} / \lambda_{q}\right) \log \mathbf{A}_{\lambda_{p}}},
$$

where $\log \left(\mathbf{A}_{\lambda_{p}}\right)$ is the matrix $\operatorname{logarithm}$ of $\mathbf{A}_{\lambda_{p}}$. This result is derived using a coupled-mode theory treatment of optical fibers to model a linear change in effective optical path length of the fiber as the wavelength is varied $[21,22]$. The full relationship between fiber TMs at different wavelengths is complex [23], so our linear approximation is limited to the "spectral bandwidth" of the fiber. The model considers a length of MMF as a sequence of infinitesimal segments that introduce field coupling between modes, $d \mathbf{m} \in \mathbb{C}^{2 M \times 1}$, such that

$$
d \mathbf{m}=d \mathbf{A} \mathbf{m},
$$

where $d \mathbf{A} \in \mathbb{C}^{2 M \times 2 M}$ represents an infinitesimal coupling matrix, and $\mathbf{m} \in \mathbb{C}^{2 M \times 1}$ represents the field in each of $M$ spatial modes over two orthogonal polarizations. This matrix equation represents a system of $2 M$ linear differential equations. The solution to these equations for any arbitrary input set of modes, $\mathbf{x}$, after traveling a distance $\ell$ along the central axis of the fiber, is given by a matrix exponential $[21,24]$ : 


$$
\mathbf{y}^{\prime}=e^{d \mathbf{A} \ell} \mathbf{x} .
$$

Since the TM $\mathbf{A}_{\lambda_{1}}=e^{d \mathbf{A} \ell_{\lambda_{1}}}$, where $\ell_{\lambda_{1}}$ is the optical path length along the central fiber axis at wavelength $\lambda_{1}$, we can obtain $d \mathbf{A}$ from the matrix logarithm of $\mathbf{A}_{\lambda_{1}}$ by

$$
d \mathbf{A}=\frac{\log \mathbf{A}_{\lambda_{1}}}{\ell_{\lambda_{1}}}
$$

Matrix logarithms, in general, produce degenerate solutions with eigenvalues of the form $\gamma+i 2 \pi n / \ell_{\lambda_{1}}, n \in \mathbb{Z}$, but because of the bandwidth-limitation imposed here, we need only consider $n=0$ (see Appendix B).

Neglecting dispersion within the relatively small (less than $10 \mathrm{~nm}$ ) spectral bandwidth, a reasonable assumption for typical glasses [25], we determine the equivalent optical path length $\ell_{\lambda_{q}}$ at the other characterization wavelengths $\left(\lambda_{q}=\lambda_{2}, \ldots, \lambda_{Q}\right)$ :

$$
\ell_{\lambda_{q}}=\frac{\lambda_{1}}{\lambda_{q}} \ell_{\lambda_{1}}
$$

Substituting Eqs. (10) and (11) into Eq. (9) produces Eq. (7) as desired.

In contrast to the zeroth-order model, when the TMs are related by this first-order model, it is not straightforward to solve for $\mathbf{A}_{\lambda_{q}}$ based on Eq. (5) applied at different wavelengths. Therefore, we present an optimization-based approach that can compute $\mathbf{A}_{\lambda_{q}}$ for $\lambda_{q}=\lambda_{1}, \ldots, \lambda_{Q}$ by repeatedly solving Eq. (5) at different wavelengths, exploiting the relationship given by Eq. (7). This approach, summarized in Algorithm 1, takes as input $R_{\lambda_{q}}$ with $\lambda_{q}=\lambda_{1}, \ldots, \lambda_{Q}$, which are measured in advance, and $C_{\lambda_{q}}$ with $\lambda_{q}=\lambda_{1}, \ldots, \lambda_{Q}$, which are measured in situ (Appendix A 2).

Algorithm 1. Recovering TMs using first-order model.

Input: $R_{\lambda_{q}}, C_{\lambda_{q}}, \lambda_{q}, q=1, \ldots, Q$

1: Solve zeroth-order model to obtain initial estimate of TM at wavelength $\lambda_{1}, \hat{\mathbf{A}}_{\lambda_{1}}$.

2: while convergence criteria not met do

3: for $\lambda_{q}=\lambda_{1}, \ldots, \lambda_{Q-1}$ do

4: $\quad$ Starting from $\hat{\mathbf{A}}_{\lambda_{q}}$, estimate $\mathrm{TM}$ at wavelength $\lambda_{q}$ : $\hat{\mathbf{A}}_{\lambda_{q}}^{\prime}=\arg \min _{\mathbf{A}}\left\|\mathbf{A}^{\top} \mathbf{R}_{\lambda_{q}} \mathbf{A}-\mathbf{C}_{\lambda_{q}}\right\|$.

5: $\quad$ Convert TM estimate to wavelength $\lambda_{q+1}$ : $\hat{\mathbf{A}}_{\lambda_{q+1}}=e^{\left(\lambda_{q} / \lambda_{q+1}\right) \log \left(\hat{\mathbf{A}}_{\lambda_{q}}^{\prime}\right)}$.

6: $\quad$ end for

7: $\quad$ Starting from $\hat{\mathbf{A}}_{\lambda_{Q-1}}$, estimate $\mathrm{TM}$ at wavelength $\lambda_{Q}$ : $\hat{\mathbf{A}}_{\lambda_{Q}}^{\prime}=\arg \min _{\mathbf{A}}\left\|\mathbf{A}^{\top} \mathbf{R}_{\lambda_{Q}} \mathbf{A}-\mathbf{C}_{\lambda_{Q}}\right\|$.

8: Convert TM estimate to wavelength $\lambda_{1}$ : $\hat{\mathbf{A}}_{\lambda_{1}}=e^{\left(\lambda_{Q} / \lambda_{1}\right) \log \left(\hat{\mathbf{A}}_{\lambda_{Q}}^{\prime}\right)}$.

9: Compute convergence criteria.

10: end while

output TM at wavelength $\lambda: \hat{\mathbf{A}}_{\lambda}=e^{\left(\lambda_{1} / \lambda\right) \log \left(\hat{\mathbf{A}}_{\lambda_{1}}^{\prime}\right)}$.
A solution to each suboptimization problem within the loop, $\hat{\mathbf{A}}_{\lambda_{q}}^{\prime}$, may be found using an iterative gradient descent solver but will not be unique because of the quadratic form of Eq. (5). By converting between wavelengths in the iterative process above, such local minima may be avoided as they are typically not minima at other wavelengths, allowing the global minimum to be found (save for a global sign error). The convergence criteria may be defined as the number of iterations or an error metric such as the relative change in gradient between iterations. Both metrics are used in this work, and for numerous simulated and experimentally measured TMs, we observe empirically that $\hat{\mathbf{A}}_{\lambda_{q}}$ reliably converges to the true value of $\mathbf{A}_{\lambda_{q}}$ for $\lambda_{q}=\lambda_{1}, \ldots, \lambda_{3}$.

\section{Physical model for imaging}

Following TM recovery, the final objective is to perform imaging. To do this, we switch the operation to a new wavelength, the imaging wavelength $\lambda_{Q+1}$, longer than the wavelengths used for TM characterization, i.e., $\lambda_{Q+1}>\lambda_{1}, \ldots, \lambda_{Q}$, in order that light may pass through the reflector stack (Sec. III A). The TM at this wavelength, $\mathbf{A}_{\lambda_{Q+1}}$, is computed from the recovered $\mathbf{A}_{\lambda_{1}}$ using Eq. (7). Considering the physical model in the context of TM characterization (Fig. 1), we first project a known illumination vector $\mathbf{x}_{\text {illum }}$ at wavelength $\lambda_{Q+1}$ onto the proximal facet of the fiber, giving, at the distal facet,

$$
\mathbf{y}_{\text {illum }}^{\prime}=\mathbf{A}_{\lambda_{Q+1}} \mathbf{x}_{\text {illum }} \text {. }
$$

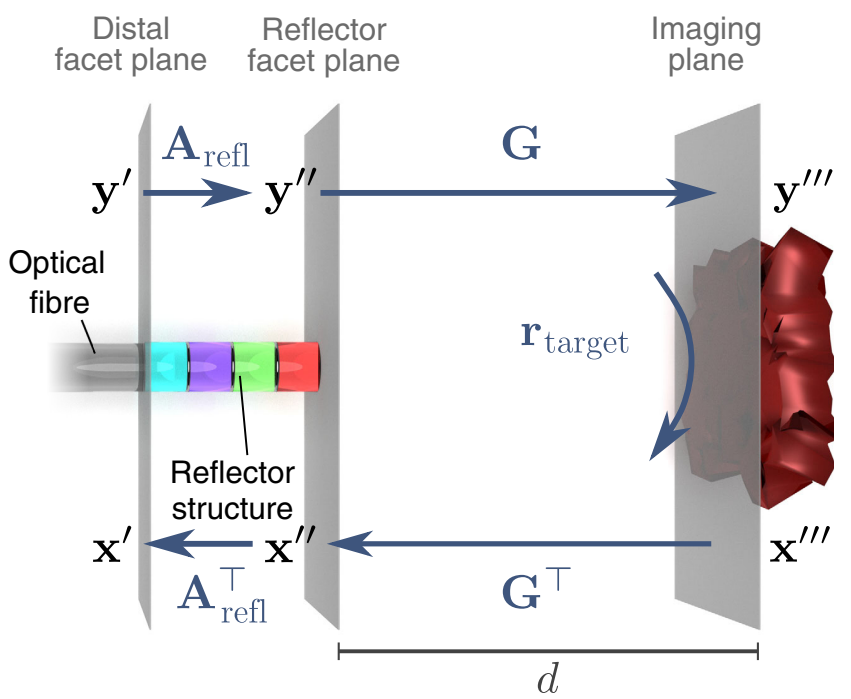

FIG. 2. Physical model used for fiber imaging. Vectors at the distal facet are denoted using the prime symbol $\left({ }^{\prime}\right)$, vectors at the exit facet of the reflector stack are denoted with double prime ("), and vectors at the imaging plane are denoted with triple prime $\left.{ }^{\prime \prime \prime \prime}\right)$. Here, $\mathbf{A}_{\text {refl }}$ represents the TM of the reflector stack, $\mathbf{G}$ is a free-space propagation operator (e.g., Fresnel diffraction parametrized by the distance $d$ between the reflector structure and the imaging plane), and $\mathbf{r}_{\text {target }}$ is the reflectance profile of the sample. 
Next, we consider the physical model from the perspective of imaging (Fig. 2), where light passes through the full reflector structure, which at wavelength $\lambda_{Q+1}$ must be partially transmissive. Denoting the TM of the reflector stack at $\lambda_{Q+1}$ by $\mathbf{A}_{\text {refl }} \in \mathbb{C}^{2 N \times 2 M}$, the illumination field exiting the reflector stack is then

$$
\mathbf{y}_{\text {illum }}^{\prime \prime}=\mathbf{A}_{\text {refl }} \mathbf{A}_{\lambda_{Q+1}} \mathbf{x}_{\text {illum }},
$$

with $\mathbf{y}_{\text {illum }}^{\prime \prime} \in \mathbb{C}^{2 N}$. Setting $N \geq M$ allows oversampled illumination fields. Note that $\mathbf{y}_{\text {illum }}^{\prime \prime}$ can be propagated by some linear operator, $\mathbf{G} \in \mathbb{C}^{2 N \times 2 N}$, through free space (e.g., Fresnel or Fraunhofer propagation) before reaching the target where the field will be

$$
\mathbf{y}_{\text {illum }}^{\prime \prime \prime}=\mathbf{G A}_{\text {refl }} \mathbf{A}_{\lambda_{Q+1}} \mathbf{x}_{\text {illum }} .
$$

Here, $\mathbf{G}$ is parametrized by the distance $d$ between the subject and the distal surface of the reflector structure (Fig. 2). In general, $d$ is not known a priori but can be estimated during operation.

Representing the target by a vector, $\mathbf{r}_{\text {target }} \in \mathbb{C}^{2 N}$, we next perform an elementwise (Hadamard) product with the illumination to give

$$
\mathbf{x}_{\text {target }}^{\prime \prime \prime}=\mathbf{y}_{\text {illum }}^{\prime \prime \prime} \circ \mathbf{r}_{\text {target }} .
$$

This light reflected from the target propagates backwards, first through free space $\mathbf{G}^{\top}$, then through the stack, $\mathbf{A}_{\text {refl }}^{\top}$, to produce a field at the distal facet:

$$
\mathbf{x}_{\text {target }}^{\prime}=\mathbf{A}_{\text {refl }}^{\top} \mathbf{G}^{\top} \mathbf{x}_{\text {target }}^{\prime \prime \prime}
$$

Next, we must also consider the illumination light that is reflected back from the reflector stack:

$$
\mathbf{x}_{\text {refl }}^{\prime}=\mathbf{R}_{\lambda_{Q+1}} \mathbf{A}_{\lambda_{Q+1}} \mathbf{x}_{\text {illum }} .
$$

We sum the two fields and propagate back through the fiber, giving

$$
\mathbf{y}_{\text {total }}=\mathbf{A}_{\lambda_{Q+1}}^{\top}\left(\mathbf{x}_{\text {target }}^{\prime}+\mathbf{x}_{\text {refl }}^{\prime}\right)
$$

as the measured quantity at the camera plane.

\section{E. Image recovery}

The goal is to recover $\mathbf{r}_{\text {target }}$ from the raw measured data $\mathbf{y}_{\text {total }}$ [Eq. (18)]. To meet this goal, we first recover $\mathbf{x}_{\text {target }}^{\prime \prime \prime}$ by rearrangement of Eq. (18) and substitution of Eqs. (16) and (17):

$$
\begin{aligned}
\left(\mathbf{A}_{\lambda_{Q+1}}^{\top}\right)^{-1} \mathbf{y}_{\text {total }}= & \left(\mathbf{x}_{\text {target }}^{\prime}+\mathbf{x}_{\text {refl }}^{\prime}\right), \\
\mathbf{x}_{\text {target }}^{\prime}= & \left(\mathbf{A}_{\lambda_{Q+1}}^{\top}\right)^{-1} \mathbf{y}_{\text {total }}-\mathbf{R}_{\lambda_{Q+1}} \mathbf{A}_{\lambda_{Q+1}} \mathbf{x}_{\text {illum }}, \\
\mathbf{x}_{\text {target }}^{\prime \prime \prime}= & \left(\mathbf{A}_{\text {refl }}^{\top} \mathbf{G}^{\top}\right)^{+}\left(\left(\mathbf{A}_{\lambda_{Q+1}}^{\top}\right)^{-1} \mathbf{y}_{\text {total }}\right. \\
& \left.-\mathbf{R}_{\lambda_{Q+1}} \mathbf{A}_{\lambda_{Q+1}} \mathbf{x}_{\text {illum }}\right),
\end{aligned}
$$

where $(\ldots)^{+}$represents a generalised inverse because $\mathbf{A}_{\text {refl }}$ may be nonsquare. Note that $\mathbf{A}_{\text {refl }}$ and $\mathbf{R}_{\lambda_{Q+1}}$ are known from prior calibration to their first use (Appendix A 2), $\mathbf{A}_{\lambda_{Q+1}}$ is estimated using Eq. (7) and $\mathbf{A}_{\lambda_{1}}$ (recovered using Algorithm 1), $\mathbf{G}$ can be estimated in postprocessing, $\mathbf{x}_{\text {illum }}$ is determined by the operator, and $\mathbf{y}_{\text {total }}$ is the measured returned field. Therefore, all quantities on the right-hand side (rhs) of Eq. (19) are known, enabling recovery of $\mathbf{x}_{\text {target }}^{\prime \prime \prime}$.

We next correct for the illumination profile incident on the target, which may resemble speckle but is deterministic. Using the model derived in Sec. III C, the noise at pixel $(x, y)$ is considered circularly symmetric, complex Gaussian distributed with standard deviation $\sigma$, so its power, $\mathrm{n}(x, y)$, is drawn from a $\chi^{2}$ distribution with 2 degrees of freedom (i.e., a Rayleigh distribution):

$$
\frac{\mathrm{n}(x, y)}{\sigma^{2}} \sim \chi^{2}(2) .
$$

Using an empirically measured value for $\sigma$, we use the $\chi^{2}$ with 2 degrees of cumulative distribution function, $\chi_{\mathrm{CDF}}^{2}(\ldots)$, to estimate for each pixel a probability that it is generated by noise, $p_{n}(x, y)$ :

$$
p_{n}(x, y)=\chi_{\mathrm{CDF}}^{2}\left(\frac{\left\|\mathbf{x}_{\text {target }}^{\prime \prime \prime}(x, y)\right\|^{2}}{\sigma^{2}}\right) .
$$

We can order $p_{n}(x, y)$ across all $(x, y)$ into a vector denoted as $\mathbf{p}_{n}$. Next, we separate the known sample illumination $\mathbf{y}_{\text {illum }}^{\prime \prime \prime}$ determined using Eq. (14), into amplitude and phase parts as

$$
\mathbf{a}_{\text {illum }}=\left|\mathbf{y}_{\text {illum }}^{\prime \prime \prime}\right|, \quad \theta_{\text {illum }}=\arg \left(\mathbf{y}_{\text {illum }}^{\prime \prime \prime}\right) .
$$

Finally, we use elementwise (Hadamard) division by a probabilistically weighted term to estimate $\tilde{\mathbf{r}}_{\text {target }}$ as follows:

$$
\tilde{\mathbf{r}}_{\text {target }}=\mathbf{x}_{\text {illum }}^{\prime \prime \prime} \oslash\left\{\left[\left(1-\mathbf{p}_{n}\right) \circ \mathbf{a}_{\text {illum }}+\mathbf{p}_{n}\right] \odot e^{i \theta_{\text {illum }}}\right\} .
$$

This approach ensures that pixels with low illumination amplitudes, and thus high noise, are not overamplified and experience only a phase correction. Pixels with larger illumination amplitudes, more likely to contain useful information, experience both amplitude and phase correction. In this way, specklelike artifacts in $\tilde{\mathbf{r}}_{\text {target }}$ are 
reduced. The full imaging process is summarized in Algorithm 2.

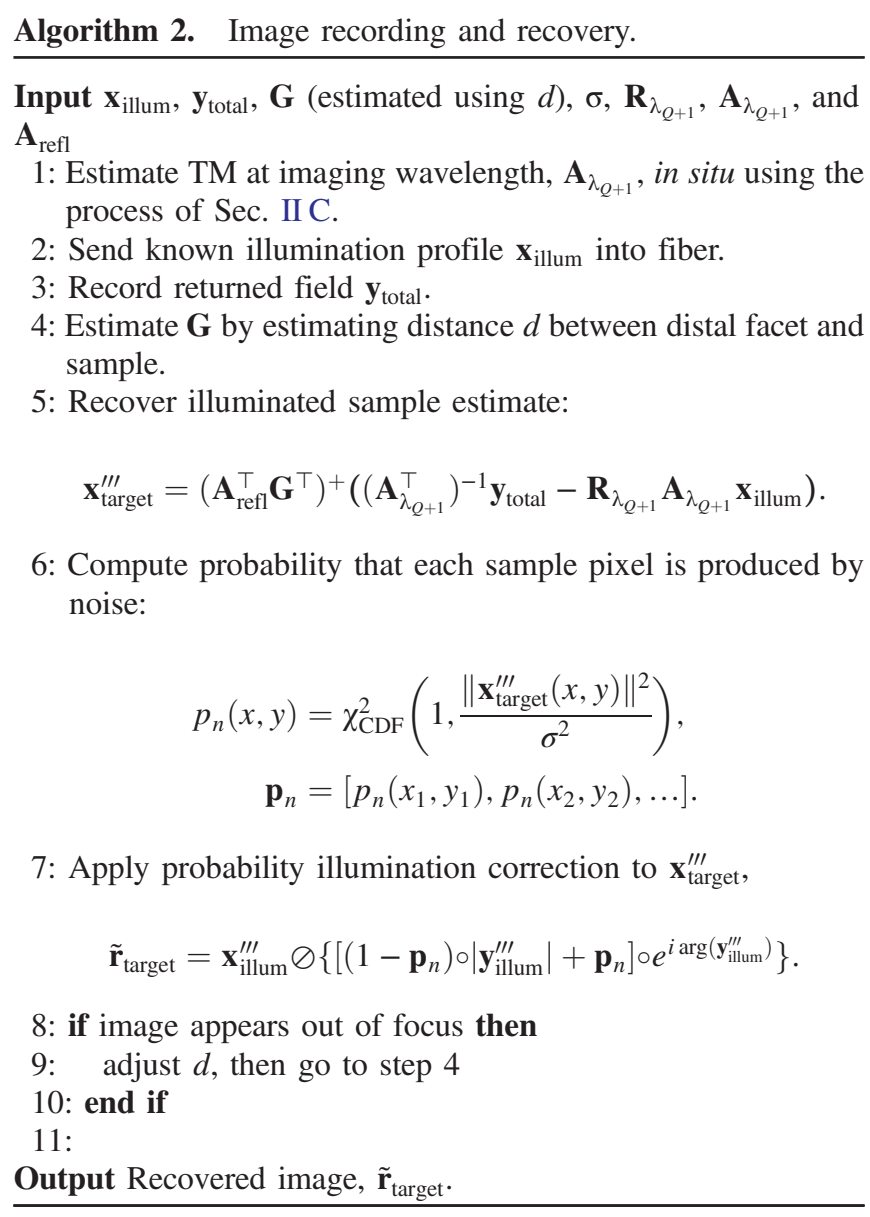

\section{METHODS}

\section{A. Implementation of reflector}

To physically achieve different PMs at different wavelengths, we propose a multilayer reflector stack concept [Fig. 3(a)]. The envisaged stack comprises layers of longpass optical filters so that light penetrates to different depths at different wavelengths [Fig. 3(b)]. Here, we use three filter layers to enable four distinct PMs - three at wavelengths used for in situ TM characterization $\left(\lambda_{1}, \lambda_{2}, \lambda_{3}\right)$ and one at the wavelength used for imaging $\left(\lambda_{4}\right)$. This method corresponds to setting $Q=3$ in Sec. II. We choose this number to minimize experimental and fabrication complexity, but knowing that $Q=1$ only admits a solution for unitary TMs [12] and finding empirically that $Q=2$ does not conclusively admit a unique solution. By contrast, the $Q=3$ case is known in the zeroth-order model to admit a unique solution (Appendix $\mathrm{C}$ ), a property that is empirically found to hold in the first-order model.

A reflector suitable for both the zeroth-order and firstorder models must couple light pseudorandomly between different modes of the fiber, including polarization modes, to create distinct RM eigenvalues with high probability (Sec. II B). To achieve this goal, we propose placing atop each filter a thin (20-50-nm) layer comprising spatially heterogeneous wire-grid polarizers [Fig. 3(c)] fabricated, for example, using electron beam lithography [17]. To act as optical polarizers, the fabricated wires must have width and pitch less than the wavelength of light $(<\lambda / 2)$, so these layers are classified as "metasurfaces." Spatial heterogeneity is achieved using arrays of partial polarizer cells that have varying linear diattenuation (or dichroism) and diattenuation axis orientations [Fig. 3(c)]. These structures can be characterized before use, for example, using the process in Appendix A 2, and are expected to remain stable throughout operation.

The optical filters ensure a different PM at each wavelength, $\mathbf{R}_{\lambda}, \lambda=\lambda_{1}, \ldots, \lambda_{4}$, because of the different sums of wavefronts occurring due to reflection and absorption from different layers [Fig. 3(a)]. Tuning a laser can then switch between reflectors without any actuation at the distal facet. The optimal wavelengths to use, ensuring maximally distinct PMs while remaining safely within the fiber's spectral bandwidth, are determined through a simple optimization process (Appendix D). Once these wavelengths are selected, the propagation of light through the multiple layers of the stack is simulated to give a physically realistic PM at each selected laser wavelength (see Appendix E).

\section{B. Fiber matrices}

For a first proof of principle, we simulated fiber TMs of size $32 \times 32$, corresponding to 16 pixels of spatial resolution in two orthogonal polarizations (Appendix F). Next, we tested experimentally measured TMs from two fibers.

The first is a MCF with TM of size $1648 \times 1648$ measured at a single wavelength of $850 \mathrm{~nm}$ [4]. This TM is used as $\mathbf{A}_{\lambda_{1}}$, and TMs at other wavelengths ( $\mathbf{A}_{\lambda_{2}}$, $\mathbf{A}_{\lambda_{3}}, \mathbf{A}_{\lambda_{4}}$ ) are generated using Eq. (7). This fiber validates that the recovery method is feasible with large TMs; however, since the TMs at other wavelengths are artificially generated, it is not a physical validation of the multiwavelength first-order model.

We therefore also tested multiwavelength experimentally measured TMs from a second fiber: a 2-m piece of stepindex MMF with $420 \times 420$ TMs measured across a wavelength range $1525-1567 \mathrm{~nm}$ in steps of $0.08 \mathrm{~nm}$ [26]. This fiber validates the multiwavelength first-order model, which is central to the scheme presented here. Because of the step-index nature of the fiber, the higherorder modes have relatively narrow spectral bandwidth (see Appendix B 2). Therefore, to improve bandwidth performance, only the 110 lowest-order modes are used, giving submatrices of size $110 \times 110$. Here, we use a wavelength spacing of $\Delta \lambda=0.9 \mathrm{~nm}$, giving TMs measured at characterization wavelengths $\lambda_{1}=1525.6 \mathrm{~nm}, \quad \lambda_{2}=$ $1526.5 \mathrm{~nm}$, and $\lambda_{3}=1527.4 \mathrm{~nm}$, and imaging wavelength 

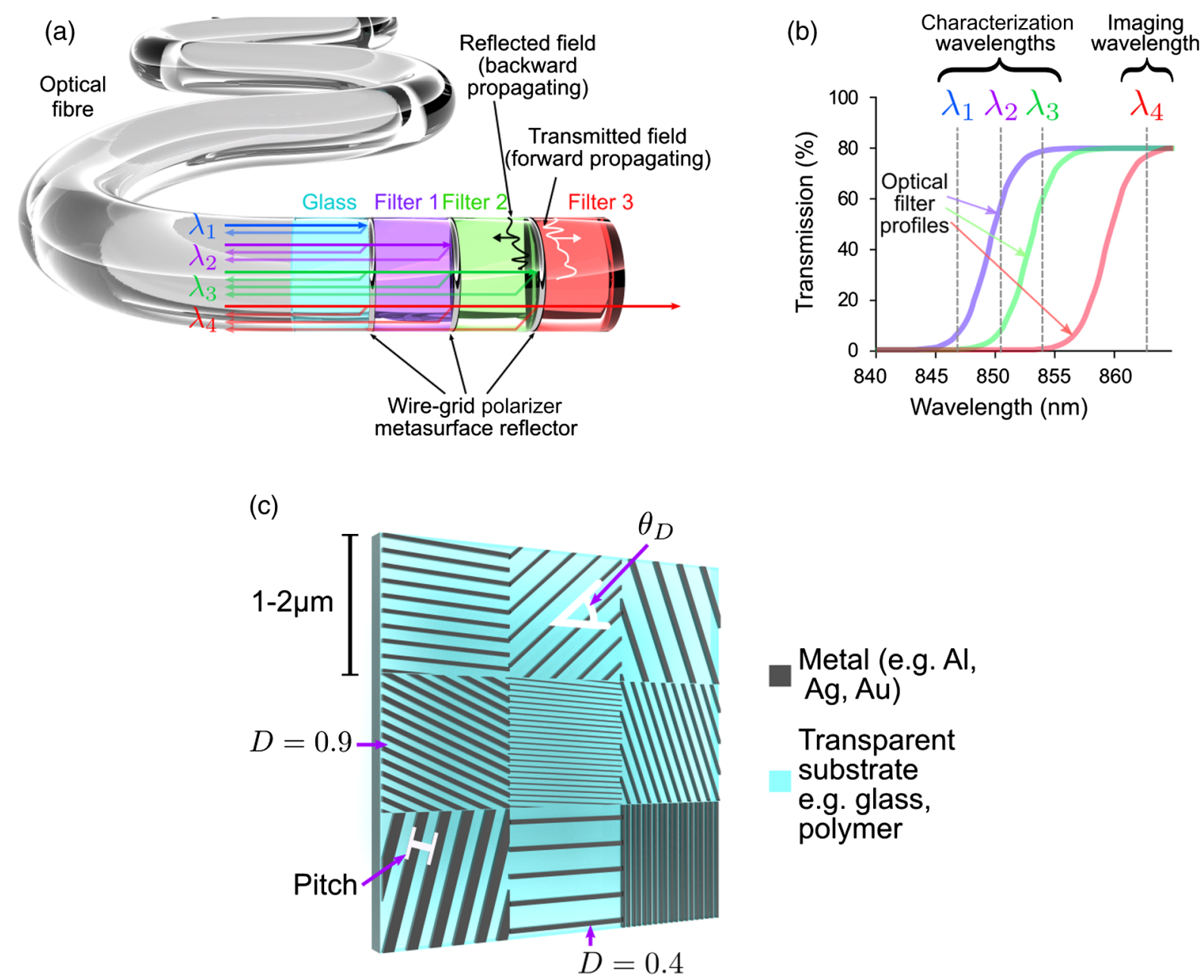

FIG. 3. Design of a wavelength-dependent reflector stack: (a) Proposed stack structure, attached to the distal facet of an optical fiber, comprising layers of glass and long-pass optical filters with wire-grid polarizer metasurfaces sandwiched between. Light paths are shown for the four different wavelengths used: the characterization wavelengths $\lambda_{1}$, $\lambda_{2}$, and $\lambda_{3}$, each producing three PMs, $\mathbf{R}_{\lambda}, \lambda=\lambda_{1}, \ldots, \lambda_{3}$; and the imaging wavelength $\lambda_{4}$ that passes through the stack. Optical fields exiting the fiber propagate through each layer via diffraction and are partially reflected at the metasurfaces generating backward propagating wavefronts. The sum of all backward propagating components represents the field coupled back into the fiber. (b) Spectral transmission profiles of the three longpass optical filters with example imaging and characterization wavelengths labeled. (c) Example design of wire-grid polarizer metasurface comprising an array of diattenuating cells of size 1-2 $\mu \mathrm{m}$ containing nanowire gratings with varying pitch (50-200 nm), duty cycle $(25 \%-75 \%)$, and orientation $(0-2 \pi)$. The pitch and duty cycle determine the linear diattenuation of each cell, $D$, and the orientation determines the diattenuation axis $\theta_{D}$. These are used to form a Jones matrix for each cell that determines its optical transmission and reflection.

$\lambda_{4}=1528.3 \mathrm{~nm}$. This wavelength separation is sufficient to practically enable the use of multiple off-the-shelf optical filters in an experimentally realized reflector stack.

The next step is to convert these TMs into RMs so that the RMs can be used to test reconstruction of the TMs, thus validating the recovery algorithm. We first simulate reflectors to produce PMs by detailed simulation of multilayer reflector stack structures, introduced in Sec. III A, assuming off-the-shelf materials (glass, standard optical filters) and randomly generated wire-grid polarizer surfaces (see Appendix E). The resulting PMs are combined with the TMs to produce RMs as per Eq. (5).
For TMs and RMs generated using the zeroth-order model, the equations of Appendix $\mathrm{C}$ are solved using code written in MATLAB. In the first-order case, the recovery algorithm (Algorithm 1) is implemented in the TensorFlow package using the GPU-optimized Adam stochastic gradient descent optimizer for improved speed [27]. The zeroth-order recovery algorithm typically finishes in less than $1 \mathrm{~s}$. However, the first-order iterative algorithm is significantly slower and can take several hours for 1500 iterations on the $1648 \times 1648$ fiber matrix on a single Tesla K40 GPU. For smaller $32 \times 32$ matrices, requiring about 500 iterations, the running time is typically less than $5 \mathrm{~s}$. 


\section{Noise model}

The relatively strong optical confinement (i.e., wave guiding) during RM characterization will result in large detected optical power and, therefore, approximately Gaussian shot noise. Laser power fluctuation (relative intensity noise) is not inherently Gaussian, but after time averaging and repeated matrix operations, it creates a Gaussian contribution to overall noise [28]. There will also be a Gaussian contribution from electronic thermal noise introduced by the image sensor. As there are many matrix operations to transform these raw images into the RMs, the repeated addition of variables (or combinations thereof) produces circularly symmetric, complex, zeromean Gaussian noise by the central limit theorem, under the assumption of independent noise between pixels [4,29].

Therefore, we model the noise in the system by adding random matrices with independent, circularly symmetric, complex Gaussian elements to each $\operatorname{PM}\left(\mathbf{R}_{\lambda_{1}}, \mathbf{R}_{\lambda_{2}}, \mathbf{R}_{\lambda_{3}}\right)$ and each RM $\left(\mathbf{C}_{\lambda_{1}}, \mathbf{C}_{\lambda_{2}}, \mathbf{C}_{\lambda_{3}}\right)$. For example, a noisy version of $\mathbf{R}_{\lambda_{3}}$ is given by

$$
\hat{\mathbf{R}}_{\lambda_{3}}=\mathbf{R}_{\lambda_{3}}+\mathbf{N}
$$

where each element of $\mathbf{N}$ is a complex random variable, $n=a+i b$, drawn from the distributions

$$
a \sim \mathcal{N}\left(0, \sigma_{n}\right), \quad b \sim \mathcal{N}\left(0, \sigma_{n}\right) .
$$

The same distributions, parametrized by $\sigma_{n}$, are used to generate noisy matrices $\hat{\mathbf{R}}_{\lambda_{1}}, \hat{\mathbf{R}}_{\lambda_{2}}, \hat{\mathbf{C}}_{\lambda_{1}}, \hat{\mathbf{C}}_{\lambda_{2}}$, and $\hat{\mathbf{C}}_{\lambda_{3}}$ for TM recovery, and noisy matrices $\hat{\mathbf{A}}_{\text {refl }}, \hat{\mathbf{R}}_{\lambda_{4}}$, and $\hat{\mathbf{y}}_{\text {total }}$ for imaging. We vary $\sigma_{n}$ over a wide range, including values reflecting empirically observed experimental noise (see Fig. 5) [4].

\section{Performance metrics}

We define several normalized error metrics to validate performance. The first quantifies the performance of the TM recovery algorithm by measuring the error between the recovered TM at the imaging wavelength, $\hat{\mathbf{A}}_{\lambda_{4}}$, and the ideal $\mathrm{TM} \mathbf{A}_{\lambda_{4}}$, given as

$$
\epsilon_{\mathrm{mat}}=\frac{\left\|\hat{\mathbf{A}}_{\lambda_{4}}-\mathbf{A}_{\lambda_{4}}\right\|}{\left\|\mathbf{A}_{\lambda_{4}}\right\|}=\frac{\left\|\left(\hat{\mathbf{A}}_{\lambda_{4}} \mathbf{A}_{\lambda_{4}}^{-1}-\mathbf{I}\right) \mathbf{A}_{\lambda_{4}}\right\|}{\left\|\mathbf{A}_{\lambda_{4}}\right\|},
$$

where $\|\ldots\|$ represents a matrix norm. A second error metric quantifies the performance of image recovery using a recovered TM:

$$
\begin{aligned}
\epsilon_{\text {mat,target }} & =\frac{\left\|\hat{\mathbf{A}}_{\lambda_{4}} \mathbf{r}_{\text {target }}-\mathbf{A}_{\lambda_{4}} \mathbf{r}_{\text {target }}\right\|_{2}}{\left\|\mathbf{A}_{\lambda_{4}} \mathbf{r}_{\text {target }}\right\|_{2}} \\
& =\frac{\left\|\left(\hat{\mathbf{A}}_{\lambda_{4}} \mathbf{A}_{\lambda_{4}}^{-1}-\mathbf{I}\right) \mathbf{A}_{\lambda_{4}} \mathbf{r}_{\text {target }}\right\|_{2}}{\left\|\mathbf{A}_{\lambda_{4}} \mathbf{r}_{\text {target }}\right\|_{2}},
\end{aligned}
$$

where $\|\ldots\|_{2}$ is the $l_{2}$ norm and $\mathbf{r}_{\text {target }}$ is the ideal image. A further metric extends the quantification of image recovery to include errors introduced in the illumination of the target:

$$
\epsilon_{\text {target }}=\frac{\left\|\hat{\mathbf{R}}_{\text {target }}-\mathbf{r}_{\text {target }}\right\|_{2}}{\left\|\mathbf{r}_{\text {target }}\right\|_{2}},
$$

where $\hat{\mathbf{R}}_{\text {target }}$ is the recovered illumination-corrected image as defined in Eq. (19).

Finally, to quantify the unitarity of a TM, $\mathbf{A}_{\lambda}$, we use the matrix condition number $\kappa\left(\mathbf{A}_{\lambda}\right)$, defined as the ratio

$$
\kappa\left(\mathbf{A}_{\lambda}\right)=\frac{\rho_{\max }\left(\mathbf{A}_{\lambda}\right)}{\rho_{\min }\left(\mathbf{A}_{\lambda}\right)}
$$

where $\rho_{\max }\left(\mathbf{A}_{\lambda}\right)$ is the largest singular value of $\mathbf{A}_{\lambda}$ and $\rho_{\min }\left(\mathbf{A}_{\lambda}\right)$ is the smallest. Therefore, the condition number of a unitary matrix is equal to 1 .

\section{RESULTS}

\section{A. Simulated TMs}

Using the first-order method (Algorithm 1), we recover a $32 \times 32$ nonunitary (condition number $\kappa=33.3$ ) simulated TM [Fig. 4(a)]. The elementwise error is less than 0.05 [Fig. 4(b)], indicating a faithful recovery of the true TM.
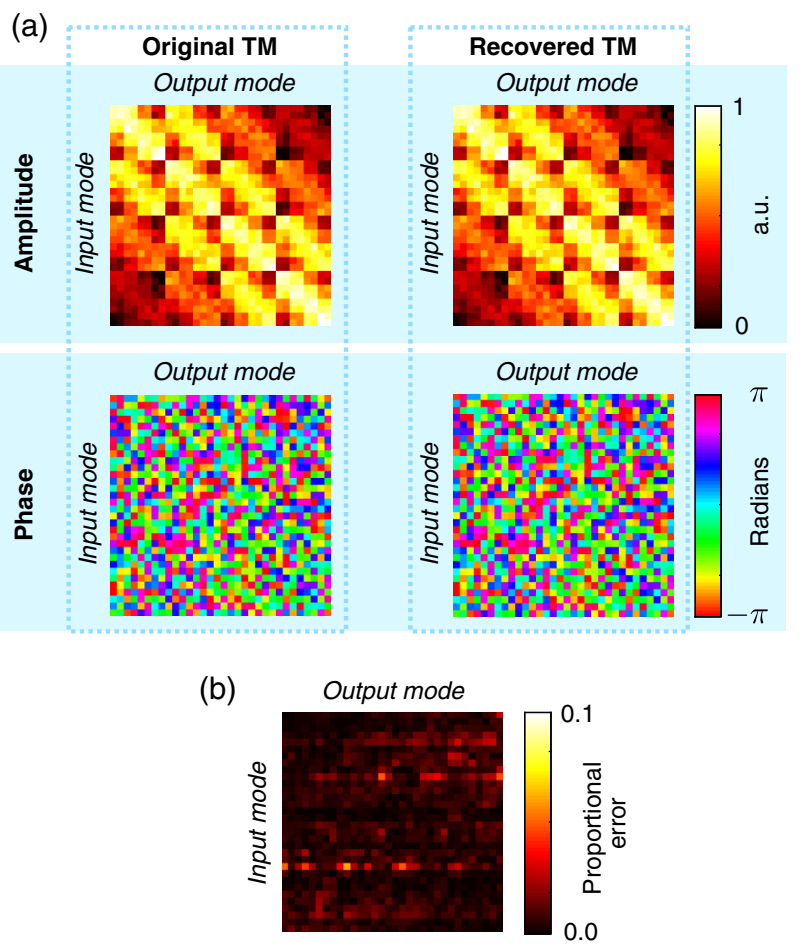

FIG. 4. Recovery of a simulated TM using the first-order recovery algorithm: (a) The original simulated TM is compared with the recovered TM (noise level $\sigma_{n}=10^{-3}$ ) demonstrating successful recovery. (b) Proportional elementwise error in TM reconstruction (maximum less than 0.05) after 300 iterations of first-order model. 
Next, we examine the impact of noise by repeating this recovery process for 64 different noise realizations with increasing noise power (see Sec. IIIC). Matrices are generated using either the zeroth- or first-order model and then reconstructed using the respective recovery algorithm. The normalized reconstruction error $\epsilon_{\text {mat,target }}$ [Eq. (24)] for the zeroth-order model deteriorates rapidly beyond a noise threshold [visualised as a "hump" in Fig. 5(a)]. In the firstorder model, which relies on an optimization process for recovery, such an instability is not observed, suggesting the
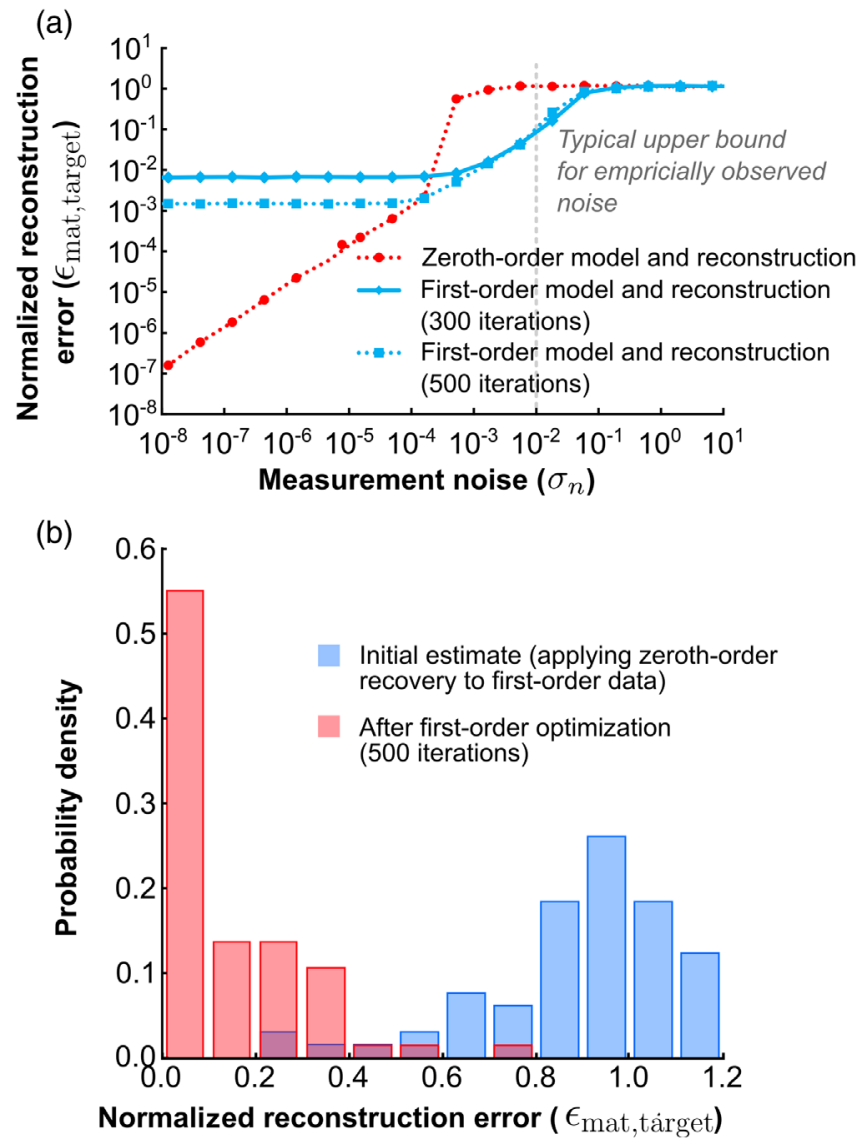

FIG. 5. Impact of measurement noise and different perturbation conditions on recovery. (a) Error in reconstructed images for different levels of noise in measured quantities ( $\epsilon_{\text {mat,target }}$ and $\sigma_{n}$ are defined in Sec. III D). At low noise levels, the error of the first-order model is seen to be limited by the number of iterations, but at higher noise levels, it converges to a trend consistent with the zeroth-order model. The first-order approach is more robust at higher noise levels due to the use of iterative optimization. A typical upper bound for noise encountered experimentally [4] is indicated to show that recovery from real experimental data is feasible. (b) Histogram showing the effect of 200 different random TM realizations, i.e., different fiber perturbations, under the first-order model. The error after the initial estimate (generated by applying zeroth-order recovery to the first-order data) can be large ( $>1$ ), but after 500 iterations of Algorithm 1, 50\% of TMs have errors less than 0.1 and $95 \%$ have errors less than 0.4 . The $\mathbf{r}_{\text {target }}$ vector used to determine $\epsilon_{\text {mat,target }}$ is a random complex unit vector. cause may be numerical error accumulation in the analytically based zeroth-order model. The first-order model exhibits improved stability at higher noise levels (such as those encountered experimentally [4]) because the optimization process explicitly seeks to minimize the effect of noise. At lower noise levels, first-order performance reaches an error plateau determined by the number of iterations.

Furthermore, testing 200 different random TM realizations, simulating possible perturbations of the fiber or manufacturing variations, shows that after 500 iterations of Algorithm 1, the error converges towards zero with greater than $50 \%$ of matrices having error less than 0.1 and $90 \%$ having error less than 0.4 [Fig. 5(b)]. Negligibly small noise power $\left(\theta_{n} \approx 0\right)$ is used so as to observe only the effect of perturbations.

\section{B. Experimentally measured MCF TM}

We next test Algorithm 1 using an experimentally measured MCF TM [4]. The raw nonsquare TM is reversibly
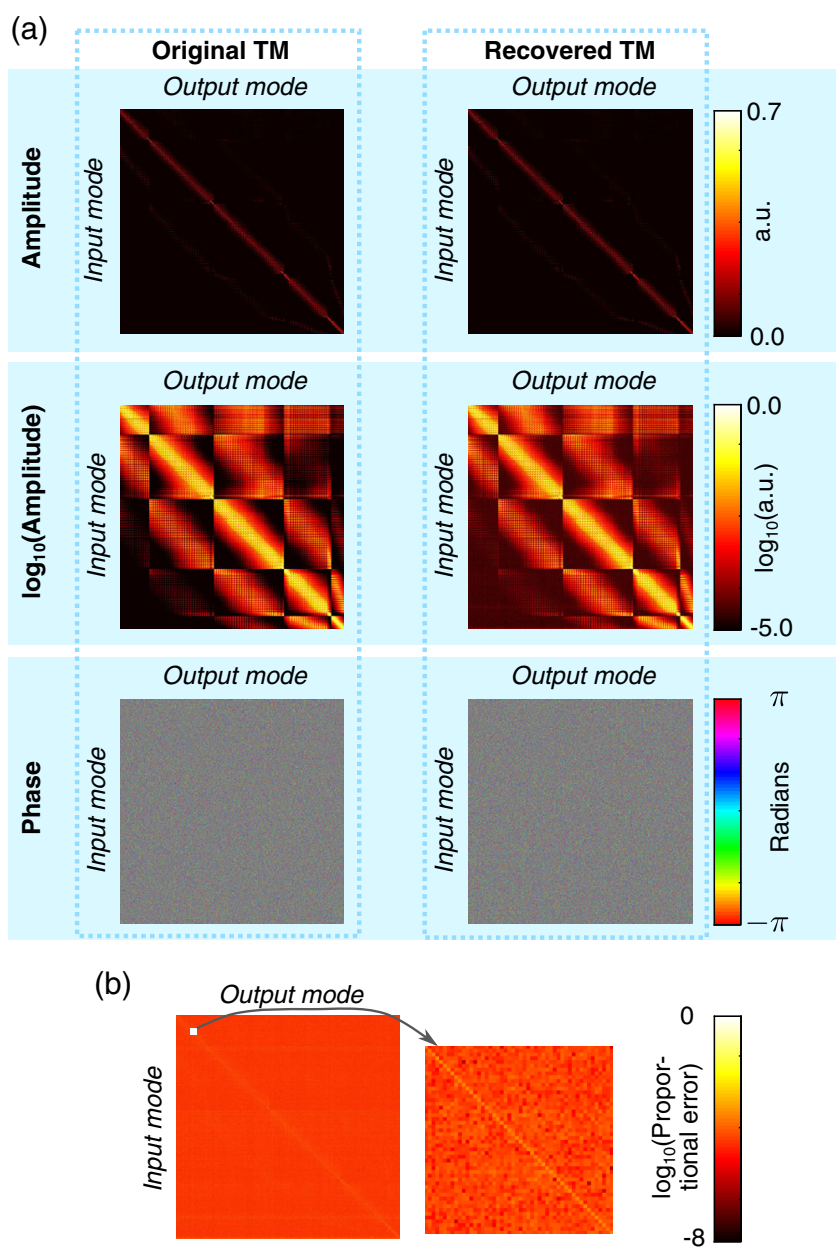

FIG. 6. First-order recovery of an experimentally measured MCF TM. (a) Original TM using data from Ref. [4] and the firstorder recovered TM, showing high visual similarity, which indicates successful recovery. (b) Proportional elementwise error in TM reconstruction (maximum less than $10^{-3}$ ). 


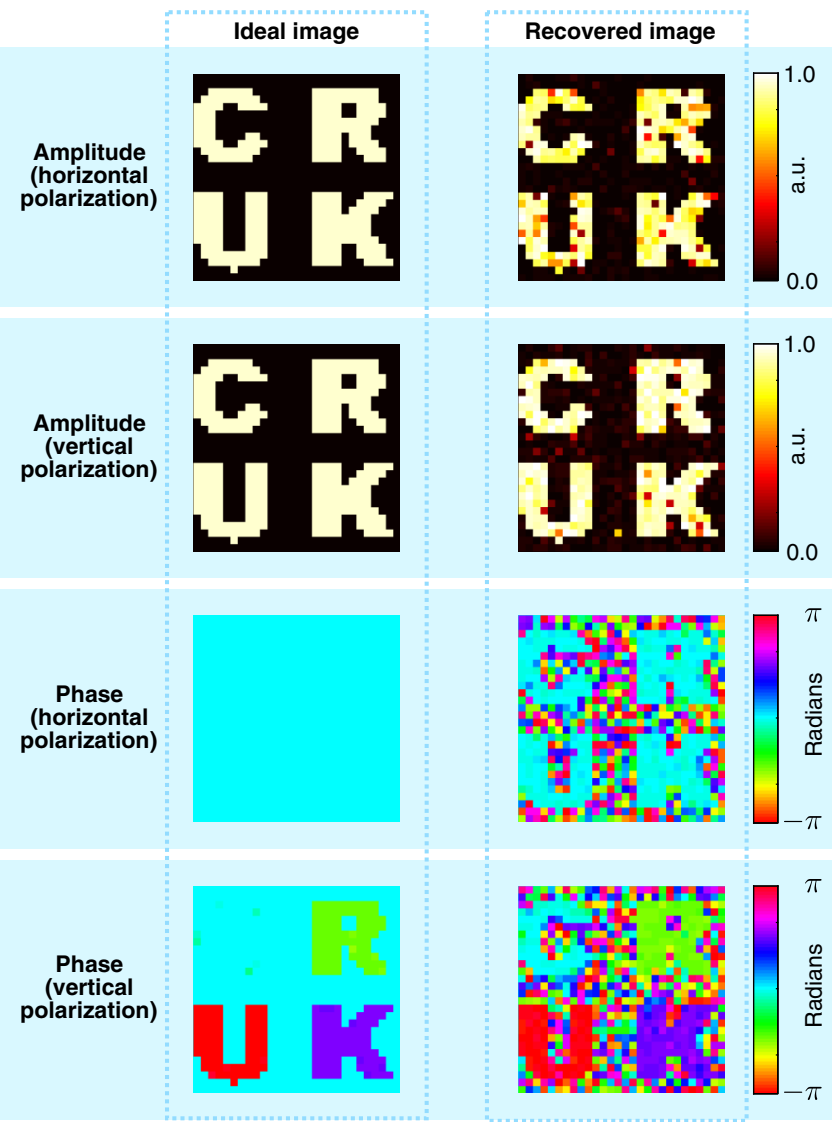

FIG. 7. Successful reconstruction of an amplitude, phase, and polarization image of a target at the distal facet using a TM recovered using the first-order model. The recovered image error is largely driven by illumination correction (see Sec. II E).

"downsampled" to a square of size $1648 \times 1648$ [Fig. 6(a)] using the process described in Appendix A 1 to form $\mathbf{A}_{\lambda_{1}}$, which has a condition number of $\kappa=3.2$. Equation (7) is then used to simulate TMs at the other wavelengths. The optimal parameters for the simulated stack (see Appendix D) are determined as follows: filters centered at $840.0 \mathrm{~nm}$, $842.7 \mathrm{~nm}$, and $849.3 \mathrm{~nm}$; characterization wavelengths of $\lambda_{1}=843.4 \mathrm{~nm}, \lambda_{2}=844.9 \mathrm{~nm}$, and $\lambda_{3}=846.5 \mathrm{~nm}$; and imaging wavelength $\lambda_{4}=850.4 \mathrm{~nm}$. Using detailed simulations of the reflector stack (Appendix E), we successfully recover the TM at the imaging wavelength $\mathbf{A}_{\lambda_{4}}$ [Fig. 6(a)]. After 1500 iterations (taking 2.1 hours), the maximum elementwise error of the recovered matrix is less than $10^{-3}$ [Fig. 6(b)]. Errors on the diagonal appear slightly exaggerated because diagonal entries of the ideal matrix (the largest magnitude entries) are used for elementwise normalization across the whole matrix.

Having recovered the TM, we next test the imaging procedure (Algorithm 2). A $28 \times 28$ image is simulated and then successfully recovered (Fig. 7). The image, $\mathbf{r}_{\text {target }}$ with reference to Fig. 2, comprises $28 x$ positions $\times 28 y$ positions $\times 2$ polarizations $=1568$ degrees of freedom (i.e., $2 N=1568$ as defined in Sec. II E), which ensures
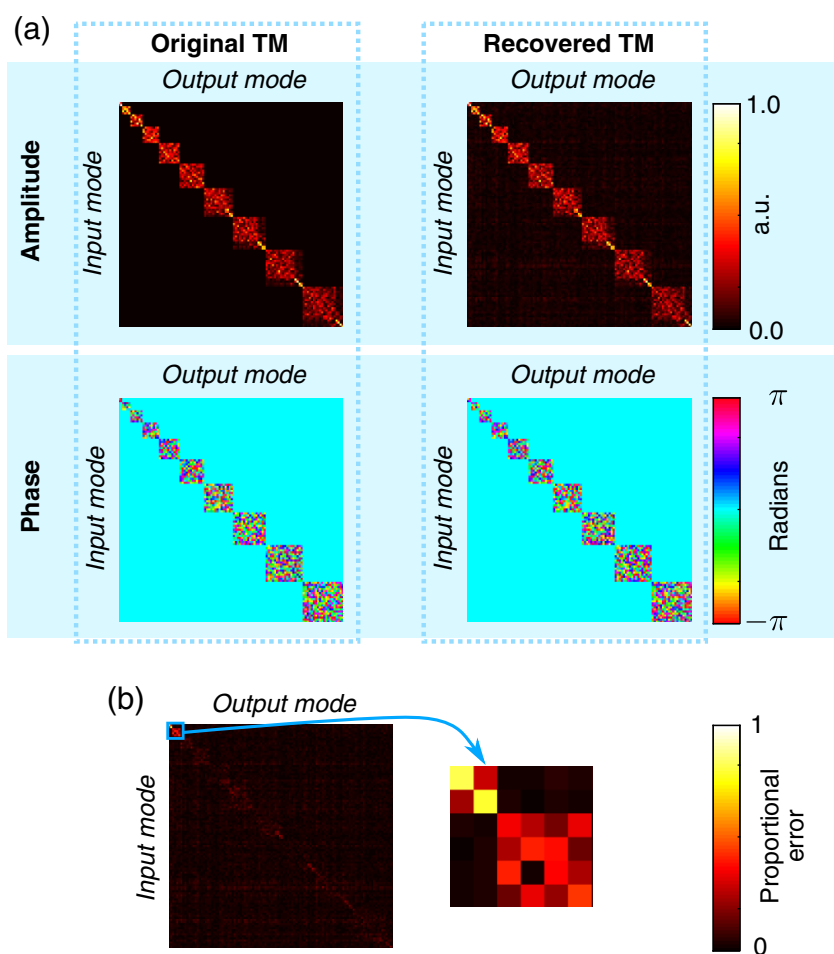

FIG. 8. First-order recovery of an experimentally measured, step-index MMF TM, using TMs recorded at multiple wavelengths (1525.6 nm, $1526.5 \mathrm{~nm}, 1527.4 \mathrm{~nm}$, and $1528.3 \mathrm{~nm}$ ) [26]. (a) Original TM using data from Ref. [26] and the first-order recovered TM, showing high visual similarity, which indicates successful recovery. (b) Proportional elementwise error in TM reconstruction values typically less than 0.05 except for the very lowest-order modes that exhibit an error of about 0.2 due to phase error.

that full reconstruction through a TM of dimension $1648 \times$ 1648 (i.e., $2 M=1648$ as defined in Sec. II E) is possible. The total target reconstruction error $\epsilon_{\text {target }}$ [Eq. (25)] is less than 0.1 and arises primarily from illumination correction. Lower illumination levels result in higher noise (see Sec. II E) and could thus be improved by averaging over multiple random illumination conditions, i.e., speckle averaging [30]. Under conditions of perfectly uniform illumination, we calculate that $\epsilon_{\text {target }}$ becomes less than 0.002 , giving a lower bound on errors dominated by other factors, e.g., computational limitations. Retrieving this image using the recovered TM takes less than $0.1 \mathrm{~s}$.

\section{Experimentally measured multiwavelength MMF TM}

Finally, we successfully demonstrate Algorithm 1 on an experimentally measured multiwavelength fiber TM data set from a 2-m piece of step-index MMF [26]. In this case, the characterization wavelengths are chosen from amongst those recorded in the data set: $\lambda_{1}=$ $1525.6 \mathrm{~nm}, \lambda_{2}=1526.5 \mathrm{~nm}$, and $\lambda_{3}=1527.4 \mathrm{~nm}$, and imaging wavelength $\lambda_{4}=1528.3 \mathrm{~nm}$. A $110 \times 110$ subset 
of the full TM data set is used in order to limit the spectral bandwidth appropriately (see Appendix B 2), and it has a condition number $\kappa=1.54$. After 475 iterations taking 14.5 minutes, the algorithm converges with significant visual similarity [Fig. 8(a)]. The matrix error $\epsilon_{\text {mat }}$ [Eq. (23)] reaches a value of 0.26. Looking at the elementwise error [Fig. 8(b)], we see that the error is typically less than 0.05 , except in the three lowest-order modes, in which the value reaches 0.2. This error is observed to manifest as phase errors, with the amplitude values being largely accurate.

\section{DISCUSSION}

The ability to form images through hair-thin optical fibers is currently limited due to the need for in situ TM calibration. To overcome this limitation, we propose a method for recovering the instantaneous TM of a fiber, based on instantaneous reflection-mode measurements combined with a priori reflection- and transmission-mode characterization of a reflector stack. Using three reflectors placed at the distal tip of the fiber, each of which produces a different reflection matrix based on a small modulation in wavelength, the imaging TM can be determined. As the fiber TM will differ slightly at each of these wavelengths, we developed a firstorder method of modeling and compensating this change using matrix exponentials, which is valid within the spectral bandwidth of the fiber. Under these assumptions, we demonstrate that it is possible to recover TMs and perform imaging using realistic optical components with acceptable noise tolerance for both simulated and measured TMs.

In particular, we demonstrate TM recovery using an experimentally measured multiwavelength TM of a stepindex MMF, validating both that the method works for real MMF and that the first-order physical model used to predict changes in TM with wavelength is physically realistic. Our findings provide the basis for in situ TM calibration and imaging through hair-thin optical fibers in living subjects. Furthermore, because the method does not place any requirements on the singular-value distribution of the TM other than that the TM is invertible, it may also be applicable to more general scattering media with very uneven distributions of singular values [31].

Nonetheless, several challenges must be overcome for experimental deployment of the proposed method. Given the demonstrated validity of the current model within the spectral bandwidth of the fiber, at $850 \mathrm{~nm}$ the wavelength modulation would need to be within about $5 \mathrm{~nm}$ for a typical 1-m length of MCF [32] or at $1550 \mathrm{~nm}$ within about $10 \mathrm{~nm}$ for a typical 2-m length of MMF [33]. In biomedical endoscopy, fiber lengths are typically 1-2 m; however, for applications in industrial inspection, greater lengths may be needed. To extend the model to work for smaller bandwidth fibers, e.g., longer fibers or MMFs with very large numbers of modes, the characterization bandwidth could be reduced. The characterization bandwidth is typically limited by the "sharpness" of available optical filters to enable significant modulations in reflectance or absorbance over very small wavelength ranges. Custom-fabricated filters for characterization may offer improved performance over off-theshelf products. Alternatively, more complex models of the fiber could be used. Complete propagation models of graded-index MMF have been used to accurately compensate bending [10], but they require precise a priori knowledge of the refractive index profile. A major advantage of the approach presented here is that no such prior knowledge of the fiber is required, enabling the use of more complex refractive index profiles such as MCF. Expanding on this, it may be possible to develop a more complex model that uses the differential changes in the TM with respect to wavelength to model the fiber over a larger bandwidth, for example, using machine learning techniques $[34,35]$.

Another challenge is speed of operation, which is determined by three components: experimental TM characterization, computational TM recovery, and image retrieval. First, for experimental TM characterization, our method uses a standard approach of sequentially projecting input fields onto the fiber facet and recording the resultant fields exiting the fiber on an image sensor. Previous works have achieved 100 input fields per second with digital micromirror devices [36] and 500 input fields per second with galvanometric scanning mirrors [30], though they are ultimately limited by camera frame rates. Our method must perform this TM characterization at three wavelengths, but it is well suited to parallelization, for example, by using multiple fixed wavelength lasers in place of a single tunable laser and multiple image sensors with dichroic filters. We therefore anticipate that future implementations of our method could achieve TM characterization speeds comparable to the state of the art in transmission-mode systems. However, further speed improvement is required for imaging live specimens that may have tissue decorrelation times as short as $50 \mathrm{~ms}$ [37]. Techniques that exploit spatial prior knowledge and sparsity to parallelize TM characterization [29] and novel high-speed cameras represent enabling steps towards real-time operation. Second, for computational TM recovery, our first-order iterative algorithm takes several minutes to recover a $110 \times 110 \mathrm{TM}$. Therefore, this algorithm requires offline reconstruction of TMs and images, although it may still prove useful in combination with a real-time imaging method used for positioning (e.g., white-light imaging through a multicore fiber). Conventional transmission-mode fiber imaging systems can avoid explicit TM recovery using the "optical phase conjugation" approach that exploits orthogonal input fields [38]. The reflection mode system here would benefit from the development of equivalent algorithms that avoid explicit TM recovery, or by replacing the iterative algorithm with an analytical solution-for example, the largely analytical, zeroth-order TM recovery algorithm presented here runs in seconds. Additional speed gains may also be 
obtained through the use of customised hardware, e.g., FPGAs. Third, because the TM is explicitly recovered, the image retrieval time is minimal $(<0.1 \mathrm{~s})$.

A final challenge is fabricating and installing an appropriate reflector stack at the distal tip of the fiber. While optical filters can be purchased off the shelf, pixellated wire-grid polarizer metasurfaces require custom fabrication using nanofabrication techniques such as electron-beam lithography. Single-layer devices of this nature have already been demonstrated academically $[17,18,39]$ and commercially [40]. Furthermore, in this application, metasurfaces can be characterized in situ, significantly relaxing fabrication tolerances. Extending fabrication to make multilayer reflector stacks will involve a number of additional deposition, lithography, and processing steps. However, the concept of using cascaded optical filters for reflection-mode Mueller matrix recovery has been experimentally validated [19]. Combined with our rigorous simulation of reflector stacks, this concept provides significant support for the feasibility of future fabrication of stacks with the required optical properties.

\section{CONCLUSION}

In summary, we have developed a new approach to determine the transmission matrix of a multimode optical fiber without requiring access to the distal facet. We have demonstrated successful transmission matrix recovery and imaging using realistic optical components with acceptable noise tolerance for simulated transmission matrices, and those experimentally measured from a multicore fiber and a multiwavelength multimode fiber. The proposed method paves the way for experimental realization of lensless imaging through hair-thin optical fibers.

Data associated with research published in this paper can be accessed at [49].

\section{ACKNOWLEDGMENTS}

G. S. D. G. acknowledges funding from Cancer Research UK (Grants No. C47594/A21102 and No. C55962/ A24669) and from a pump-priming award from the Cancer Research UK Cambridge Centre Early Detection Programme (Grant No. A20976). C. W. acknowledges funding from a Cancer Research UK Pioneer Award (Grant No. C55962/A24669). M. G. acknowledges funding from Engineering and Physical Sciences Research Council for the Centre for Mathematical and Statistical Analysis of Multimodal Clinical Imaging (Grant No. EP/N014588/1). R. P. M. acknowledges funding from the Engineering and Physical Sciences Research Council (Grant No. EP/ L015889/1). S. E. B. acknowledges funding from Cancer Research UK (Grants No. C47594/A16267, No. C14303/ A17197, and No. C47594/A21102) and the EU FP7 Agreement (Grant No. FP7-PEOPLE-2013-CIG-630729). G. S. D. G. would like to thank Peter Christopher from the University of Cambridge for useful discussions.

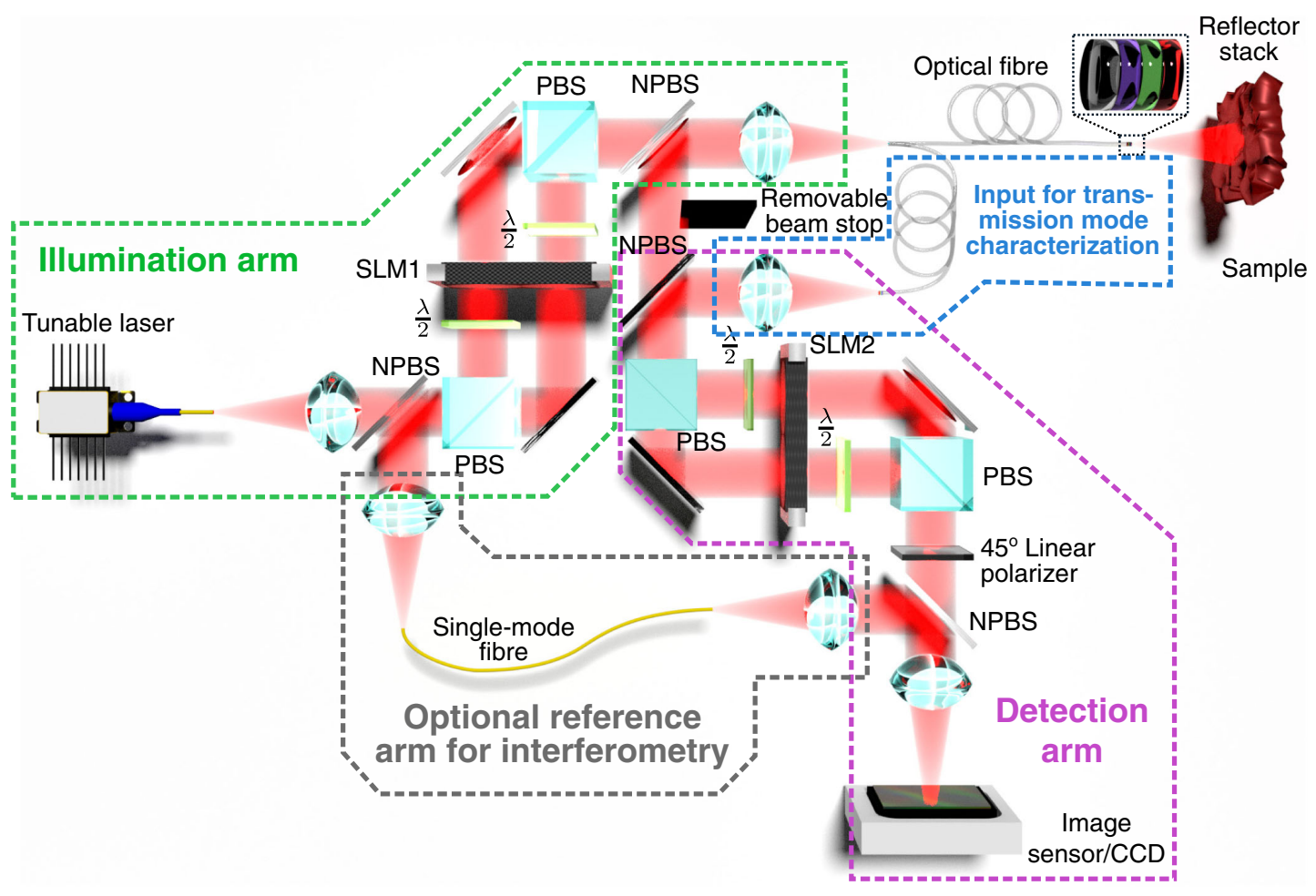

FIG. 9. Conceptual experimental setup for fiber TM characterization in the reflection mode configuration using a distal reflector stack. SLM: spatial light modulator; PBS: polarizing beam splitter; NPBS: nonpolarizing beam splitter; $\lambda / 2$ : half-wave plate. 


\section{APPENDIX A: CONCEPTUAL EXPERIMENTAL IMPLEMENTATION}

\section{Downsampling reflection matrices}

Algorithm 1 uses matrix exponentials to account for the effect of small wavelength modulations on fiber TMs. Matrix exponentials are defined only for square matrices, but in practical implementations, we may measure RMs that are nonsquare, denoted $\tilde{\mathbf{C}}_{\lambda}$ with $\lambda=\lambda_{1}, \ldots, \lambda_{4}$, due to different sampling schemes for $\mathbf{x}$ and $\mathbf{y}$ of Fig. 1. For example, the number of camera pixels often greatly exceeds the number of input calibration fields [4]. In this scenario, consider $2 M$ calibration samples $\left(\tilde{\mathbf{x}}_{s}, \tilde{\mathbf{y}}_{s}\right)_{s=1, \ldots, 2 M}$, where $\tilde{\mathbf{x}}_{s} \in \mathbb{C}^{P \times 1}$ and $\tilde{\mathbf{y}}_{s} \in \mathbb{C}^{Q \times 1}$. Denoting $\tilde{\mathbf{C}}_{\lambda} \in \mathbb{C}^{Q \times P}$ as a mapping of the fiber $\tilde{\mathbf{y}}=\tilde{\mathbf{C}}_{\lambda} \tilde{\mathbf{x}}$ (with reference to Fig. 1),

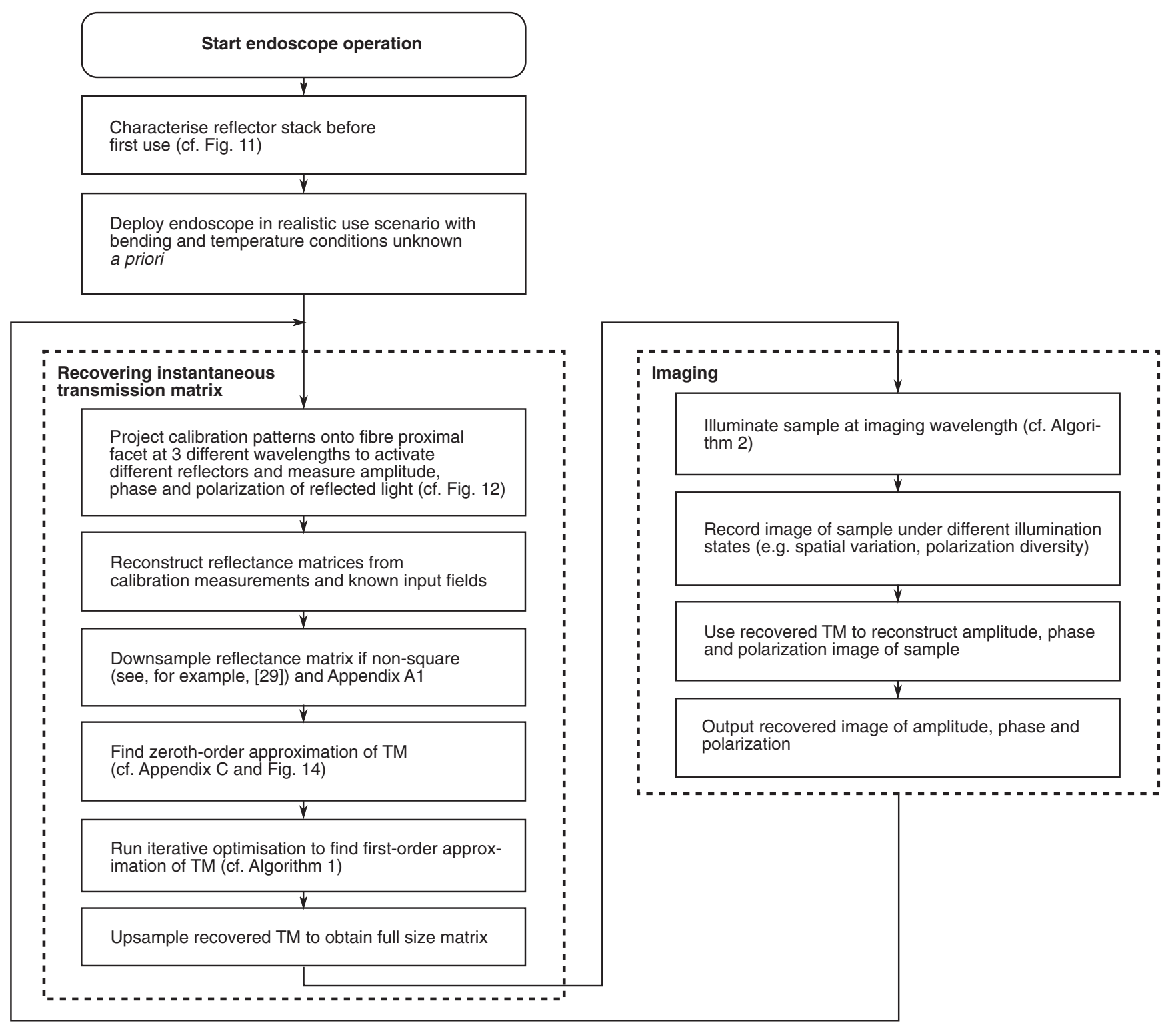

FIG. 10. Flowchart detailing the full process of TM recovery and imaging using the reflection mode endoscope setup. Details of specific subtasks are given in figures and sections as indicated. we wish to determine a downsampling process to obtain a square $\mathrm{RM}, \tilde{\tilde{\mathbf{C}}}_{\lambda} \in \mathbb{C}^{2 M \times 2 M}$, which may be used in our recovery algorithms.

One way of determining this process is to estimate the largest singular values and corresponding left singular vectors of $\tilde{\mathbf{C}}_{\lambda} \in \mathbb{C}^{Q \times P}$, which should match those of $\tilde{\tilde{\mathbf{C}}}_{\lambda} \in \mathbb{C}^{2 M \times 2 M}$. We let

$$
\begin{gathered}
\tilde{\mathbf{X}}_{\text {cal }}:=\left[\tilde{\mathbf{x}}_{1}, \ldots, \tilde{\mathbf{x}}_{2 M}\right] \in \mathbb{C}^{P \times 2 M}, \\
\tilde{\mathbf{Y}}_{\text {cal }}:=\left[\tilde{\mathbf{y}}_{1}, \ldots, \tilde{\mathbf{y}}_{2 M}\right] \in \mathbb{C}^{Q \times 2 M}
\end{gathered}
$$

so that $\tilde{\mathbf{Y}}_{\text {cal }}=\tilde{\mathbf{C}}_{\lambda} \tilde{\mathbf{X}}_{\text {cal }}$. If the coefficients of an input signal $\tilde{\mathbf{x}} \in \mathbb{C}^{P \times 1}$ with respect to the $2 M$-dimensional calibration basis are denoted by $\tilde{\mathbf{x}}_{\text {cal }} \in \mathbb{C}^{2 M \times 1}$, i.e., $\tilde{\mathbf{x}}:=\tilde{\mathbf{X}}_{\text {cal }} \tilde{\mathbf{x}}_{\text {cal }}$, then 
its output signal is given as $\tilde{\mathbf{y}}=\tilde{\mathbf{C}}_{\lambda} \tilde{\mathbf{x}}=\tilde{\mathbf{C}}_{\lambda} \tilde{\mathbf{X}}_{\text {cal }} \tilde{\mathbf{x}}_{\text {cal }}=$ $\tilde{\mathbf{Y}}_{\text {cal }} \tilde{\mathbf{x}}_{\text {cal }}$. In other words, $\tilde{\mathbf{Y}}_{\text {cal }}$ is a mapping of the fiber from the $2 M$-dimensional calibration basis to $\mathbb{C}^{Q \times 1}$, and our hope is that the largest $2 M$ singular values and

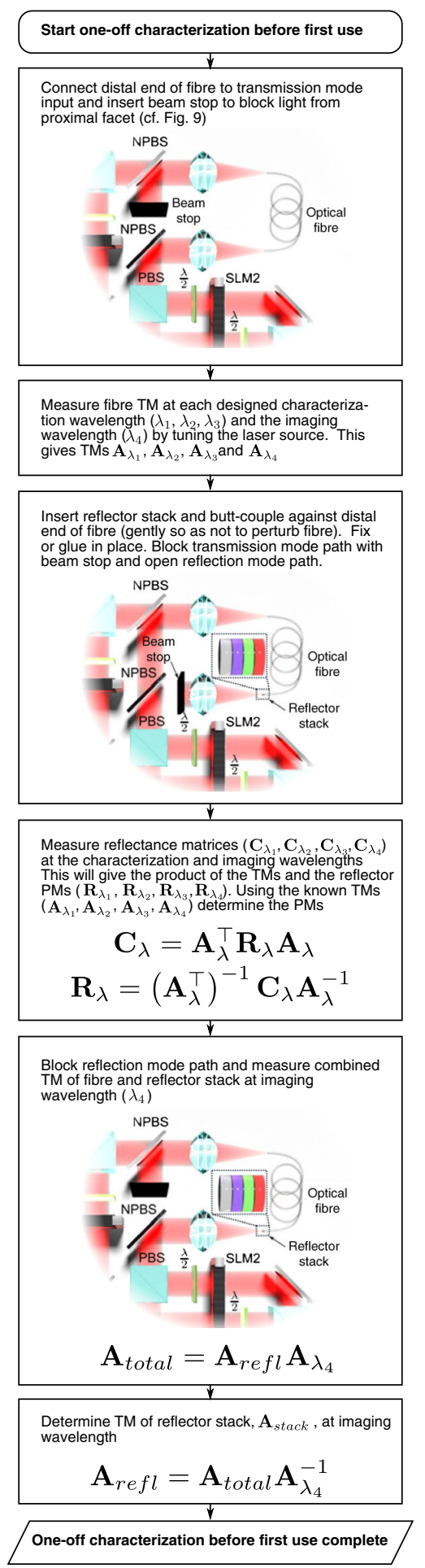

FIG. 11. Flowchart detailing the measurement process required to accurately characterize the reflector matrices of the reflector stack at different wavelengths prior to the first use of the system. This process is achieved using the experimental setup of Fig. 9. corresponding left singular vectors of $\tilde{\mathbf{Y}}_{\text {cal }}$ approximate those of $\tilde{\mathbf{C}}_{\lambda}$ and $\tilde{\tilde{\mathbf{C}}}_{\lambda}$. We therefore first truncate the singular values and right vectors of $\tilde{\mathbf{C}}_{\lambda}$ to produce $\tilde{\mathbf{C}}_{\lambda}^{\prime} \in \mathbb{C}^{Q \times 2 M}$. Next, we further compress the matrix $\tilde{\mathbf{C}}_{\lambda}^{\prime}$ into a square matrix $\tilde{\tilde{\mathbf{C}}}_{\lambda} \in \mathbb{C}^{2 M \times 2 M}$, which preserves the same singular values and left singular vectors. This can be done via a process such as that described in Ref. [29]. Following this process, $\tilde{\tilde{\mathbf{C}}}_{\lambda}$ is square, so it can be used for TM recovery as described in Secs. II B and II C.

This method enables a factorization of $\tilde{\mathbf{C}}_{\lambda}$ into the product of $\tilde{\tilde{\mathbf{C}}}_{\lambda} \in \mathbb{C}^{2 M \times 2 M}$ and the downsampling matrix $\mathbf{T} \in \mathbb{C}^{P \times 2 M}$ such that $\tilde{\mathbf{C}}_{\lambda}=\mathbf{T} \tilde{\tilde{\mathbf{C}}}_{\lambda}$. Once the $\mathrm{TM}$ has been determined, $\mathbf{T}$ can be used to reconstruct $\tilde{\mathbf{C}}_{\lambda}$ if desired.

\section{Proposed experimental design}

A conceptual experimental setup for reflection-mode TM recovery is shown in Fig. 9. There are two key sections: the illumination arm and the detection arm. These perform similar functions to previous work [4] but are both located at the proximal fiber facet. There is also an additional input that allows characterization in the transmission mode prior

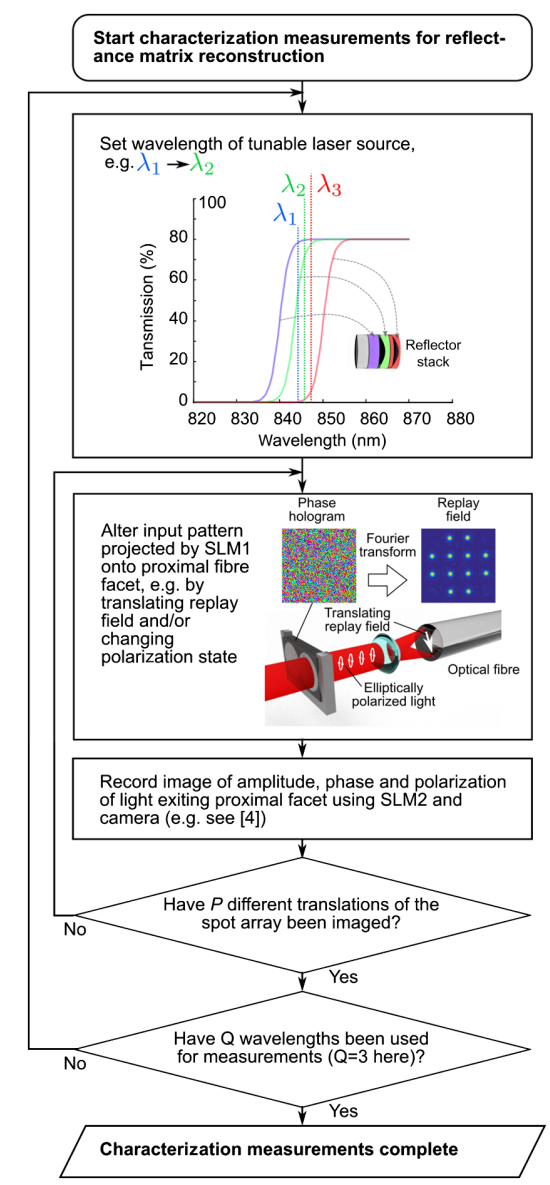

FIG. 12. Flowchart detailing the series of measurements required to recover instantaneous RMs using the setup of Fig. 9. 
to use. The tunable laser is required to provide a highcoherence source at the different characterization and imaging wavelengths. These can typically be tuned in steps of $0.1-0.2 \mathrm{~nm}$, sufficient to cover a range of fiber TMs with different spectral bandwidths. A parallelized design would have the same basic structure but with several multiplexed fixed wavelength lasers and several image sensors multiplexed by dichroic filters.

Figure 10 shows a flow chart for the full operating process, using the setup of Fig. 9, for recovering TMs and performing imaging.

Before using the system for the first time, we must take accurate measurements of the reflector stack RMs at all wavelengths and its TM at the imaging wavelength. A flowchart detailing this process is given in Fig. 11. Nonsquare measured TMs $\tilde{\mathbf{A}}_{\lambda}$ and RMs $\tilde{\mathbf{C}}_{\lambda}$ with $\lambda=$ $\lambda_{1}, \ldots, \lambda_{4}$ may, if needed, be downsampled to form square approximations as described in Appendix A 1.

The sequence of experimental measurements required to record instantaneous RMs at each respective wavelength $\left[\mathbf{C}_{\lambda}, \lambda=\lambda_{1}, \ldots, \lambda_{4}\right.$ of Eq. (5)] is illustrated in Fig. 12.

\section{APPENDIX B: SPECTRAL BANDWIDTH}

\section{Phase change within spectral bandwidth}

Let us call the center wavelength of the fiber $\lambda_{0}$ and the spectral bandwidth of the fiber $\Delta \lambda_{\text {fib }}$. The bandwidth in frequency units is

$$
\begin{aligned}
\Delta f=f_{1}-f_{2} & =\frac{c}{n_{\mathrm{eff}}\left(\lambda_{0}-\Delta \lambda_{\mathrm{fib}} / 2\right)}-\frac{c}{n_{\mathrm{eff}}\left(\lambda_{0}+\Delta \lambda_{\mathrm{fib}} / 2\right)} \\
& =\frac{c}{n_{\mathrm{eff}}}\left(\frac{1}{\lambda_{0}-\Delta \lambda_{\mathrm{fib}} / 2}-\frac{1}{\lambda_{0}+\Delta \lambda_{\mathrm{fib}} / 2}\right),
\end{aligned}
$$

where $n_{\text {eff }}$ is the effective refractive index of the fiber (e.g., around 1.5 for glass) and $c$ is the speed of light in a vacuum. The time-of-flight difference for the longest path lengths is given by

$$
\Delta t=\frac{1}{\Delta f}=\frac{n_{\mathrm{eff}}}{c} \frac{1}{\left(\frac{1}{\lambda_{0}-\Delta \lambda_{\mathrm{fib}} / 2}-\frac{1}{\lambda_{0}+\Delta \lambda_{\mathrm{fib}} / 2}\right)} .
$$

A fiber with physical length $\ell_{1}$ will produce a shortest time of flight across all modes of approximately

$$
t_{1}=\frac{\ell_{1}}{\frac{c}{n_{\text {eff }}}}
$$

and therefore a longest time of flight of

$$
t_{2}=t_{1}+\Delta t
$$

This case results in an effective optical path length for the longest path of

$$
\begin{aligned}
\ell_{2} & =\frac{c}{n_{\mathrm{eff}}} t_{2}=\frac{c}{n_{\mathrm{eff}}}\left(t_{1}+\Delta t\right)=\frac{c}{n_{\mathrm{eff}}}\left(\frac{\ell}{\frac{c}{n_{\mathrm{eff}}}}+\Delta t\right) \\
& =\ell+\frac{c}{n_{\mathrm{eff}}} \Delta t, \\
\ell_{2} & =\ell_{1}+\frac{1}{\left(\frac{1}{\lambda_{0}-\Delta \lambda_{\mathrm{fib}} / 2}-\frac{1}{\lambda_{0}+\Delta \lambda_{\mathrm{fib}} / 2}\right)} .
\end{aligned}
$$

At the shortest test wavelength, $\lambda_{0}-\Delta \lambda / 2$, the phase shift introduced by the shortest path is

$$
\phi_{1, \text { short }}=\frac{2 \pi \ell}{\lambda_{0}-\Delta \lambda / 2},
$$

and that introduced by the longest path is

$\phi_{1, \text { long }}=\frac{2 \pi \ell_{2}}{\lambda_{0}-\Delta \lambda / 2}=\frac{2 \pi\left(\ell+\frac{1}{\left(\frac{1}{\lambda_{0}-\Delta \lambda_{\mathrm{fb}} / 2}-\frac{1}{\lambda_{0}+\Delta \lambda_{\mathrm{fb}} / 2}\right)}\right)}{\lambda_{0}-\Delta \lambda / 2}$,

giving a difference of

$$
\begin{aligned}
\Delta \phi_{1} & =\phi_{1, \text { long }}-\phi_{1, \text { short }}=2 \pi \frac{\frac{1}{\left(\frac{1}{\lambda_{0}-\Delta \lambda_{\mathrm{fib}} / 2}-\frac{1}{\lambda_{0}+\Delta \lambda_{\mathrm{fib}} / 2}\right)}}{\lambda_{0}-\Delta \lambda / 2} \\
& =2 \pi \frac{1}{\left(\frac{1}{\lambda_{0}-\Delta \lambda_{\mathrm{fib}} / 2}-\frac{1}{\lambda_{0}+\Delta \lambda_{\mathrm{fbb}} / 2}\right)\left(\lambda_{0}-\Delta \lambda / 2\right)} .
\end{aligned}
$$

At the longest test wavelength, $\lambda_{0}+\Delta \lambda / 2$, the phase shift introduced by the shortest path is

$$
\phi_{2, \text { short }}=\frac{2 \pi \ell}{\lambda_{0}+\Delta \lambda / 2},
$$

and that introduced by the longest path is

$\phi_{2, \text { long }}=\frac{2 \pi \ell_{2}}{\lambda_{0}+\Delta \lambda / 2}=\frac{2 \pi\left(\ell+\frac{1}{\left(\frac{1}{\lambda_{0}-\Delta \lambda_{\mathrm{fib}} / /}-\frac{1}{\lambda_{0}+\Delta \lambda_{\mathrm{fib}} / 2}\right)}\right)}{\lambda_{0}+\Delta \lambda / 2}$,

giving a difference of

$$
\begin{aligned}
\Delta \phi_{2} & =\phi_{2, \text { long }}-\phi_{2, \text { short }}=2 \pi \frac{\frac{1}{\left(\frac{1}{\lambda_{0}-\Delta \lambda_{\text {fib }} / 2}-\frac{1}{\lambda_{0}+\Delta \lambda_{\text {fib }} / 2}\right)}}{\lambda_{0}+\Delta \lambda / 2} \\
& =2 \pi \frac{1}{\left(\frac{1}{\lambda_{0}-\Delta \lambda_{\text {fib }} / 2}-\frac{1}{\lambda_{0}+\Delta \lambda_{\text {fib }} / 2}\right)\left(\lambda_{0}+\Delta \lambda / 2\right)} .
\end{aligned}
$$

The difference in phase shift between the longest and shortest paths at the two test wavelengths is then

$$
\begin{aligned}
\Delta \phi_{1}-\Delta \phi_{2}= & \frac{2 \pi}{\left(\frac{1}{\lambda_{0}-\Delta \lambda_{\mathrm{fib}} / 2}-\frac{1}{\lambda_{0}+\Delta \lambda_{\mathrm{fib}} / 2}\right)} \\
& \times\left(\frac{1}{\lambda_{0}-\Delta \lambda / 2}-\frac{1}{\lambda_{0}+\Delta \lambda / 2}\right),
\end{aligned}
$$


which after rearrangement becomes

$$
2 \pi \frac{\Delta \lambda}{\Delta \lambda_{\text {fib }}} \frac{\lambda_{0}^{2}-\left(\Delta \lambda_{\mathrm{fib}} / 2\right)^{2}}{\lambda_{0}^{2}-(\Delta \lambda / 2)^{2}} .
$$

We observe that for small test bandwidths $\Delta \lambda$, this phase shift tends to zero. Thereafter, the phase shift monotonically increases with $\Delta \lambda$. When the test bandwidth reaches the fiber bandwidth $\left(\Delta \lambda=\Delta \lambda_{\text {fib }}\right)$, the phase shift reaches $2 \pi$, thus demonstrating that if the wavelength is kept within the spectral bandwidth, the phase shift introduced between any two paths will be less than or equal to $2 \pi$. This result can be inferred intuitively: If path lengths vary in phase by significantly less than $2 \pi$ when the wavelength is varied, the diffracted far-field pattern will remain similar, so the "speckle correlation" will be high [41].

\section{Bandwidth of step-index MMF}

To validate the first-order recovery algorithm, we use a data set of TMs taken at multiple wavelengths (1525$1567 \mathrm{~nm}$ in steps of $0.08 \mathrm{~nm}$ ) from a step refractive index profile MMF with 420 modes (including polarization modes) [26]. To examine the bandwidth over which the first-order model is valid, we first consider a singlewavelength TM at some reference wavelength $\lambda_{\text {ref }}$, denoted $\mathbf{A}_{\mathrm{MMF}}\left(\lambda_{\text {ref }}\right)$. Using Eq. (7), we then estimate the singlewavelength TM at another test wavelength, $\lambda_{\text {test }}>\lambda_{\text {ref }}$, as

$$
\mathbf{A}_{\text {est }}\left(\lambda_{\text {test }}, \lambda_{\text {ref }}\right)=e^{\left[\left(\lambda_{\text {ref }} / \lambda_{\text {test }}\right) \log \mathbf{A}_{\mathrm{MMF}}\left(\lambda_{\text {ref }}\right)\right]} .
$$

Next, we define an error metric to test the predictive accuracy of the first-order model:

$\epsilon_{\text {est }}\left(\lambda_{\text {test }}, \lambda_{\text {ref }}\right)=\sqrt{\frac{\left\|\mathbf{A}_{\text {est }}\left(\lambda_{\text {test }}, \lambda_{\text {ref }}\right)-\mathbf{A}_{\mathrm{MMF}}\left(\lambda_{\text {test }}\right)\right\|}{\left\|\mathbf{A}_{\mathrm{MMF}}\left(\lambda_{\text {test }}\right)\right\|}}$,

where $\mathbf{A}_{\mathrm{MMF}}\left(\lambda_{\text {test }}\right)$ is the measured MMF TM at wavelength $\lambda_{\text {test }}$ and $\|\ldots\|$ is a matrix norm. Defining $\Delta \lambda=\lambda_{\text {test }}-\lambda_{\text {ref }}$, we can rewrite Eq. (B14) as

$\epsilon_{\text {est }}(\Delta \lambda)=\sqrt{\frac{\left\|\mathbf{A}_{\text {est }}\left(\Delta \lambda, \lambda_{\text {ref }}\right)-\mathbf{A}_{\mathrm{MMF}}\left(\lambda_{\text {ref }}+\Delta \lambda\right)\right\|}{\left\|\mathbf{A}_{\mathrm{MMF}}\left(\lambda_{\text {ref }}+\Delta \lambda\right)\right\|}}$.

Taking $\lambda_{\text {ref }}$ as $1526 \mathrm{~nm}$, we can then plot $\epsilon_{\text {est }}$ as a function of $\Delta \lambda$ (Fig. 13). We find empirically that changing $\lambda_{\text {ref }}$ does not significantly change this curve.

The full $420 \times 420$ matrix provides $\epsilon_{\text {est }}=0.5$ for a $\Delta \lambda$ of about $0.5 \mathrm{~nm}$. An appropriate reflector stack design for this fiber would require three filters with center wavelengths less than $0.1 \mathrm{~nm}$ apart, which is challenging using only offthe-shelf components. To increase the available bandwidth, we restrict our analysis to the lowest-order modes of the fiber and form an $N \times N$ submatrix comprising the first $N$ columns of the first $N$ rows of each $\mathbf{A}_{\mathrm{MMF}}$. The resulting

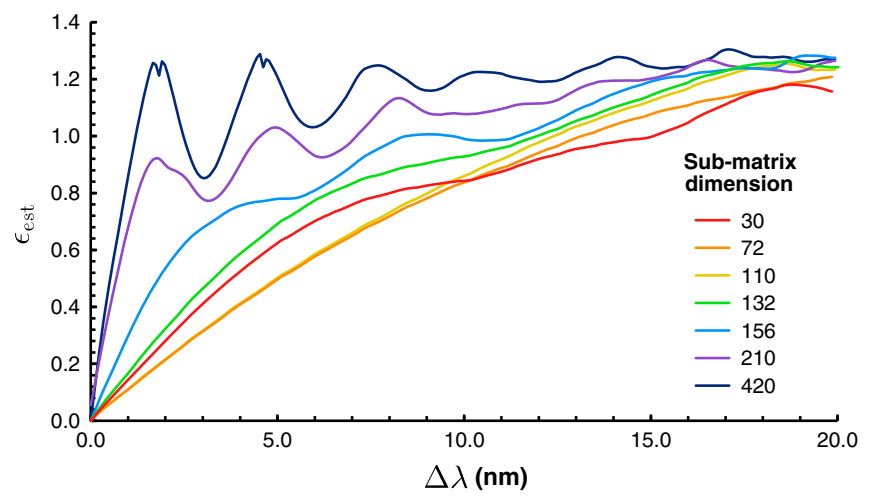

FIG. 13. Validation of the first-order model of Appendix A 2 using measured multiwavelength TMs from a step-index MMF [26]. The normalized error of the TM predicted by the first-order model compared with the actual measured TM $\left(\epsilon_{\text {est }}\right)$ is plotted as the difference in reference and test wavelengths $(\Delta \lambda)$ is increased. Different submatrices of only the lowest-order modes are considered: that is, submatrices of size $N \times N$, with $N=30$, $72,110,132,156,210,420$. For lower-order submatrices, the first-order model is seen to be valid over a larger wavelength range.

$\epsilon_{\text {est }}$ curves for $N=30,72,110,132,156,210,420$ are plotted in Fig. 13. We find that using the 110 lowest-order modes gives a $\Delta \lambda$ of $5 \mathrm{~nm}$ for $\epsilon_{\text {est }}=0.5$, ample to design a filter stack using off-the-shelf components.

In reality, dispersion will further change the effective optical path lengths at the different wavelengths. From the data set here, we compute that, over a 5-nm range, the error introduced is less than $3 \%$ and is therefore neglected in this work.

\section{APPENDIX C: ZEROTH-ORDER MODEL}

\section{Derivation of solution}

Using the zeroth-order assumption implied by Eq. (6), we consider the application of Eq. (5) at three wavelengths (i.e., $Q=3$ ) to obtain

$$
\begin{aligned}
\mathbf{C}_{\lambda_{1}} & =\mathbf{A}^{\top} \mathbf{R}_{\lambda_{1}} \mathbf{A}, \\
\mathbf{C}_{\lambda_{2}} & =\mathbf{A}^{\top} \mathbf{R}_{\lambda_{2}} \mathbf{A}, \\
\mathbf{C}_{\lambda_{3}} & =\mathbf{A}^{\top} \mathbf{R}_{\lambda_{3}} \mathbf{A} .
\end{aligned}
$$

Starting from Eqs. (C1) and (C2), we derive

$$
\mathbf{C}_{\lambda_{2}}^{-1} \mathbf{C}_{\lambda_{1}}=\mathbf{A}^{-1} \mathbf{R}_{\lambda_{2}}^{-1} \mathbf{R}_{\lambda_{1}} \mathbf{A}
$$

which we compactly write as

$$
\mathbf{C}_{\alpha}=\mathbf{A}^{-1} \mathbf{R}_{\alpha} \mathbf{A}
$$

where $\mathbf{C}_{\alpha}=\mathbf{C}_{\lambda_{2}}^{-1} \mathbf{C}_{\lambda_{1}} \in \mathbb{C}^{2 M \times 2 M}, \mathbf{R}_{\alpha}=\mathbf{R}_{\lambda_{2}}^{-1} \mathbf{R}_{\lambda_{1}} \in \mathbb{C}^{2 M \times 2 M}$. We observe that $\mathbf{C}_{\alpha}$ and $\mathbf{R}_{\alpha}$ are similar matrices, and they 
have the same eigenvalues. Equation $(\mathrm{C} 4)$ is then transformed into

$$
\mathbf{R}_{\alpha} \mathbf{A}-\mathbf{A C}_{\alpha}=\mathbf{0} .
$$

Here, $\mathbf{R}_{\lambda_{1}}$ and $\mathbf{R}_{\lambda_{2}}$ can be designed so as to avoid the trivial solution $\mathbf{A}-\mathbf{A}=\mathbf{0}$ arising if $\mathbf{R}_{\alpha}$ and $\mathbf{C}_{\alpha}$ are both identity matrices. Similarly, we derive, from Eqs. (C2) and (C3),

$$
\mathbf{R}_{\beta} \mathbf{A}-\mathbf{A C}_{\beta}=\mathbf{0},
$$

where $\mathbf{C}_{\beta}=\mathbf{C}_{\lambda_{3}}^{-1} \mathbf{C}_{\lambda_{2}}$ and $\mathbf{R}_{\beta}=\mathbf{R}_{\lambda_{3}}^{-1} \mathbf{R}_{\lambda_{2}}$, and from Eqs. (C1) and (C3),

$$
\mathbf{R}_{\gamma} \mathbf{A}-\mathbf{A C}_{\gamma}=\mathbf{0},
$$

where $\mathbf{C}_{\gamma}=\mathbf{C}_{\lambda_{3}}^{-1} \mathbf{C}_{\lambda_{1}}$ and $\mathbf{R}_{\gamma}=\mathbf{R}_{\lambda_{3}}^{-1} \mathbf{R}_{\lambda_{1}}$. Equations (C5)(C7) are examples of Sylvester equations, and since we know $\mathbf{A} \neq \mathbf{0}$, as this would represent a system with $100 \%$ power loss, we can use established methods to determine the space of nontrivial solutions. Specifically, we implement the Bartels-Stewart algorithm for solving Sylvester equations of the form $\mathbf{D X}-\mathbf{X E}=\mathbf{F}$ for $\mathbf{X}$ in the special case where $\mathbf{F}=\mathbf{0}$ [42].

The first step of the Bartels-Stewart algorithm uses the Schur matrix decomposition, which states that any square matrix $\mathbf{H}$ can be decomposed as

$$
\mathbf{H}=\mathbf{Q U Q}^{-1},
$$

where $\mathbf{Q}$ is unitary and $\mathbf{U}$ is an upper triangular matrix [43]. Importantly, the diagonal of $\mathbf{U}$ comprises the eigenvalues of $\mathbf{H}$ sorted in descending order of magnitude. With reference to Eq. (C5), we can then write

$$
\begin{aligned}
\mathbf{R}_{\alpha} & =\mathbf{Q}_{R_{\alpha}} \mathbf{U}_{R_{\alpha}} \mathbf{Q}_{R_{\alpha}}^{-1}, \\
\mathbf{C}_{\alpha} & =\mathbf{Q}_{C_{\alpha}} \mathbf{U}_{C_{\alpha}} \mathbf{Q}_{C_{\alpha}}^{-1} .
\end{aligned}
$$

Substituting Eqs. (C9) and (C10) back into Eq. (C5) gives

$$
\begin{array}{r}
\mathbf{Q}_{R_{\alpha}} \mathbf{U}_{R_{\alpha}} \mathbf{Q}_{R_{\alpha}}^{-1} \mathbf{A}-\mathbf{A} \mathbf{Q}_{C_{\alpha}} \mathbf{U}_{C_{\alpha}} \mathbf{Q}_{C_{\alpha}}^{-1}=\mathbf{0}, \\
\mathbf{U}_{R_{\alpha}} \mathbf{Q}_{R_{\alpha}}^{-1} \mathbf{A} \mathbf{Q}_{C_{\alpha}}-\mathbf{Q}_{R_{\alpha}}^{-1} \mathbf{A} \mathbf{Q}_{C_{\alpha}} \mathbf{U}_{C_{\alpha}}=\mathbf{0}, \\
\mathbf{U}_{R_{\alpha}} \mathbf{A}^{\prime}-\mathbf{A}^{\prime} \mathbf{U}_{C_{\alpha}}=\mathbf{0},
\end{array}
$$

where

$$
\mathbf{A}^{\prime}=\mathbf{Q}_{R_{\alpha}}^{-1} \mathbf{A} \mathbf{Q}_{C_{\alpha}}
$$

and $\mathbf{A}^{\prime} \in \mathbb{C}^{2 M \times 2 M}$. Equation (C11) is another Sylvester equation; however, crucially, $\mathbf{U}_{R_{\alpha}}$ and $\mathbf{U}_{C_{\alpha}}$ are upper triangular matrices, so we can solve for $\mathbf{A}^{\prime}$ element by element as is typically done in the Bartels-Stewart algorithm. By suitable design of the reflectors $\mathbf{R}_{\lambda_{1}}$ and $\mathbf{R}_{\lambda_{2}}$ (randomly generated matrices will suffice $[44,45]$ ), the eigenvalues of $\mathbf{R}_{\alpha}$ (and hence $\mathbf{C}_{\alpha}, \mathbf{U}_{R_{\alpha}}$, and $\mathbf{U}_{C_{\alpha}}$ since they are similar matrices) can be made distinct. Consequently, it follows that only the diagonal elements of $\mathbf{A}^{\prime}$ are indeterminate and that the elements of $\mathbf{A}^{\prime}$ are related to elements of $\mathbf{U}_{C_{\alpha}}$ and $\mathbf{U}_{R_{\alpha}}$ as

$$
\begin{aligned}
& {\left[u r_{P, Q} a_{Q, Q}-\sum_{s=P}^{Q-1} u c_{s, Q} a_{P, s}\right]+\left(u r_{P, P}-u c_{Q, Q}\right) a_{P, Q}} \\
& \quad+\sum_{q=P+1}^{Q-1} u r_{P, q} a_{q, Q}=0
\end{aligned}
$$

where $u c_{P, Q}$ represents an element of $\mathbf{U}_{C_{\alpha}}$ at row $P$ and column $Q, u r_{P, Q}$ represents an element of $\mathbf{U}_{R_{\alpha}}$ at row $P$ column $Q$, and $a_{P, Q}$ represents an element of $\mathbf{A}^{\prime}$ at row $P$ and column $Q$ (see Appendix C 2 for a full derivation).

Varying $P$ from 1 to $Q-1$ in Eq. (C13) produces $Q-1$ equations. If we arbitrarily set the diagonal elements, $a_{m, m}$, of $\mathbf{A}^{\prime}$, we are left with $Q-1$ unknowns and can solve this system of linear equations for all elements of the $Q$ th column of $\mathbf{A}^{\prime}$. By varying $Q$ from 2 to $2 M$ and solving each resultant system of equations, we can fully determine all nondiagonal elements of $\mathbf{A}^{\prime}$ given a set of arbitrary diagonal elements, $a_{m, m}(m=1, \ldots, 2 M)$. This case can be expressed as a single matrix equation, but it requires an $M(2 M-1) \times M(2 M-1)\left(\approx 4 M^{4}\right.$ element $)$ matrix, which becomes computationally intractable as $M$ grows.

When an arbitrary set of $2 M$ complex numbers (i.e., a vector $\in \mathbb{C}^{2 M}$ ) is selected for the diagonal elements of $\mathbf{A}^{\prime}$ in Eq. (C11), the remaining elements of $\mathbf{A}^{\prime}$ can be solved. This case implies that the "true" solution for $\mathbf{A}^{\prime}$ can be expressed as a linear combination of, at most, $2 M$ matrices, $\mathbf{A}_{m}^{\prime}$, where $m=1, \ldots, 2 M$, each of which is a solution of Eq. (C11). Together, these $2 M$ matrices comprise a basis for $\mathbf{A}^{\prime}$ [equivalently, they comprise a null space of Eq. (C11)]. Using Eq. (C12), this basis can be converted to a basis for $\mathbf{A}$ [equivalently, a null space for Eq. (C5)], denoted $\tilde{\mathbf{A}}_{m}$ where $m=1, \ldots, 2 M$. To improve robustness to noise and numerical error, an iterative Gram-Schmidt orthogonalization procedure can be applied to create an orthogonal set of $\tilde{\mathbf{A}}_{m}$, where $m=1, \ldots, 2 M$. The process of determining a basis for $\mathbf{A}$ is summarized in Fig. 14.

Having determined a suitable basis, we express the matrix we wish to recover, $\mathbf{A}$, as

$$
\mathbf{A}=w_{1} \tilde{\mathbf{A}}_{1}+\ldots+w_{2 M} \tilde{\mathbf{A}}_{2 M},
$$

where $w_{m}$ represents the complex-valued weight of the $m$ th basis element, the matrix $\tilde{\mathbf{A}}_{m}$. The solution space now has only $2 M$ degrees of freedom (represented by $w_{m}$ ), so computational complexity can be further reduced by considering a subset of elements of $\mathbf{A}$. We can select $B(\geq 2 M)$ 

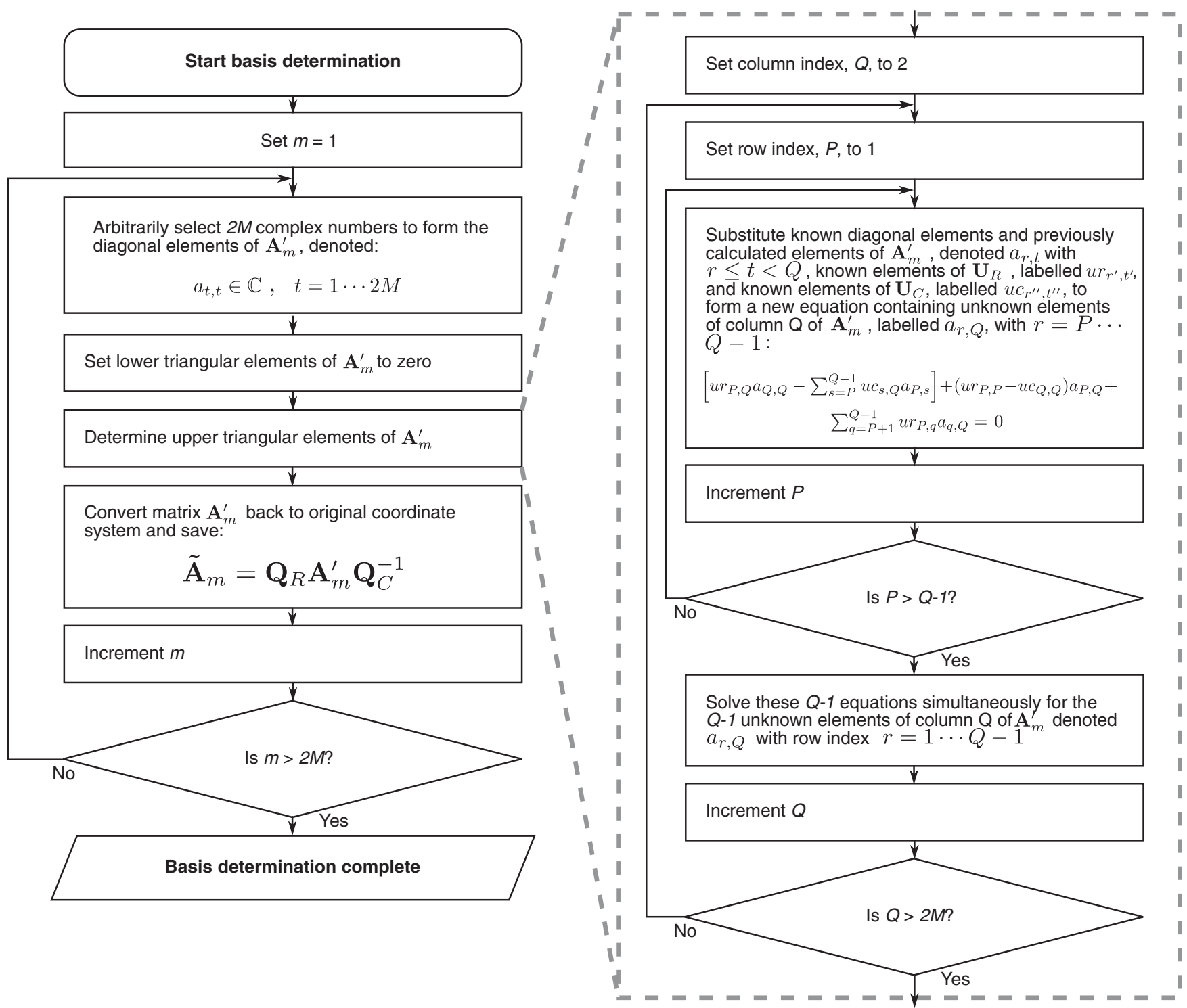

FIG. 14. Flowchart detailing the process of finding a basis for the matrix $\mathbf{A}$ using the zeroth-order solution method.

matrix elements of $\mathbf{A}$, either arbitrarily or according to some prior information, e.g., the elements with the largest mean based on a statistical model. The corresponding $B$ elements of $\tilde{\mathbf{A}}_{m}$ are then ordered into a column vector $\mathbf{b}_{m} \in$ $\mathbb{C}^{B}$ for every $m=1, \ldots, 2 M$. These column vectors form a matrix $\mathbf{B}_{\alpha} \in \mathbb{C}^{B \times 2 M}$ :

$$
\mathbf{B}_{\alpha}=\left[\begin{array}{llll}
\mathbf{b}_{1} & \mathbf{b}_{2} & \cdots & \mathbf{b}_{2 M}
\end{array}\right],
$$

so we can write

$$
\mathbf{B}_{\alpha} \mathbf{w}^{\top}=\mathbf{b}_{\mathrm{est}},
$$

where $\mathbf{b}_{\text {est }}$ is an estimate of the $B$ selected elements of the true TM, $\mathbf{A}$, and $\mathbf{w}=\left[w_{1} \cdots w_{N}\right]$ is a vector containing the complex weights of Eq. (C14). Since $\mathbf{B}_{\alpha}$ is either a square or tall matrix, we premultiply by its Moore-Penrose pseudo-inverse, $\mathbf{B}_{\alpha}^{\dagger}$ :

$$
\mathbf{B}_{\alpha}^{\dagger} \mathbf{B}_{\alpha} \mathbf{w}^{\top}=\mathbf{B}_{\alpha}^{\dagger} \mathbf{b}_{\text {est }}, \quad \mathbf{w}^{\top}=\mathbf{B}_{\alpha}^{\dagger} \mathbf{b}_{\text {est }} .
$$

We then multiply both sides by $\mathbf{B}_{\alpha}$ to get

$$
\mathbf{B}_{\alpha} \mathbf{w}^{\top}=\mathbf{B}_{\alpha} \mathbf{B}_{\alpha}^{\dagger} \mathbf{b}_{\mathrm{est}}
$$

and substitute this in Eq. (C16) to obtain a recursive expression:

$$
\mathbf{b}_{\mathrm{est}}=\mathbf{B}_{\alpha} \mathbf{B}_{\alpha}^{\dagger} \mathbf{b}_{\mathrm{est}} .
$$

Following the same steps to derive Eq. (C17) from Eq. (C5) but starting from Eq. (C6) gives 


$$
\mathbf{b}_{\mathrm{est}}=\mathbf{B}_{\beta} \mathbf{B}_{\beta}^{\dagger} \mathbf{b}_{\mathrm{est}} .
$$

Similarly, starting from Eq. (C7) gives

$$
\mathbf{b}_{\mathrm{est}}=\mathbf{B}_{\gamma} \mathbf{B}_{\gamma}^{\dagger} \mathbf{b}_{\mathrm{est}} \cdot
$$

Clearly, $\mathbf{b}_{\text {est }}$ is an eigenvector of $\mathbf{B}_{\alpha} \mathbf{B}_{\alpha}^{\dagger}, \mathbf{B}_{\beta} \mathbf{B}_{\beta}^{\dagger}$, and $\mathbf{B}_{\gamma} \mathbf{B}_{\gamma}^{\dagger}$ with an eigenvalue of 1 . Physical considerations of power conservation suggest the existence of at least one nontrivial solution for $\mathbf{b}_{\text {est }}$. Our empirical investigations with both simulated and real TMs (see Sec. IV) suggest that when using at least two of Eqs. (C17)-(C19), we can identify a single nontrivial solution, the "true" solution: We arbitrarily choose Eqs. (C17) and (C18). Next, we apply a variant of the "power method" for finding dominant eigenvalues of a matrix [46] and recursively substitute Eq. (C17) into Eq. (C18), and substitute the result into Eq. (C17), etc., to give

$$
\mathbf{b}_{\text {est }}^{t}=\underbrace{\mathbf{B}_{\alpha} \mathbf{B}_{\alpha}^{\dagger} \mathbf{B}_{\beta} \mathbf{B}_{\beta}^{\dagger} \ldots \mathbf{B}_{\alpha} \mathbf{B}_{\alpha}^{\dagger}}_{\text {product of } t+1 \text { matrices }} \mathbf{b}_{\mathrm{est}}^{0},
$$

where $t$ denotes the number of matrix multiplications. For large $t$, the output $\mathbf{b}_{\text {est }}^{t}$ is expected to converge to the desired solution $\mathbf{b}_{\text {est }}$ for any input $\mathbf{b}_{\text {est }}^{0}$. Alternatively, for large $t$, the dominant eigenvector of $\mathbf{B}_{\alpha} \mathbf{B}_{\alpha}^{\dagger} \mathbf{B}_{\beta} \mathbf{B}_{\beta}^{\dagger} \ldots \mathbf{B}_{\alpha} \mathbf{B}_{\alpha}^{\dagger}$ will approximate the solution well. In this work, we find empirically that using 2-3 iterations, i.e., $t \in\{3,5\}$, is sufficient for reliable TM recovery.

To obtain the desired full TM A, we determine the weights $\mathbf{w}$ from approximation $\mathbf{b}_{\text {est }}^{t}$ by solving Eq. (C16) and then computing the appropriate weighted sum of $\mathbf{A}_{m}$, with $m=1, \ldots, 2 M$ using Eq. (C14).

\section{Sylvester equations element by element using Bartels-Stewart algorithm}

Starting from Eq. (C11), we wish to solve for matrix $\mathbf{A}^{\prime}$ element by element. Recalling that all matrices here are $\in \mathbb{C}^{N \times N}$, we first consider the element in row $N$, column 1 , i.e., $(N, 1)$, of the zero matrix on the rhs of Eq. (C11). We see that

$$
u r_{N, N} a_{N, 1}-a_{N, 1} u c_{1,1}=0,
$$

where $u r_{m, n}$ is the element in row $m$, column $n$ of $\mathbf{U}_{R_{\alpha}}$; $u c_{m, n}$ is the element in row $m$, column $n$ of $\mathbf{U}_{C_{\alpha}}$; and $a_{m, n}$ is the element in row $m$, column $n$ of $\mathbf{A}^{\prime}$.

Next, we require that the eigenvalues of $\mathbf{R}_{\alpha}$ are distinct, which is ensured by a suitable design of reflectors. We also know that the eigenvalues of $\mathbf{R}_{\alpha}$ are equal to those of $\mathbf{C}_{\alpha}$ because the two are similar matrices [see Eq. (C4)]. Therefore, the values on the diagonal of $\mathbf{U}_{R_{\alpha}}$ are distinct from one another, and they are equal to the values on the diagonal of $\mathbf{U}_{C_{\alpha}}$, i.e., $u r_{m, m}=u c_{m, m}$ for every $m$. The only way that Eq. (C21) can hold is if $a_{N, 1}=0$, providing the solution for that element.

We then consider element $(N-1,1)$, giving

$$
u r_{N-1, N-1} a_{N-1,1}+u r_{N-1, N} a_{N, 1}-a_{N-1,1} u c_{1,1}=0 .
$$

Since we know that $a_{N, 1}=0$, we again apply the above reasoning and conclude that $a_{N-1,1}=0$. Continuing up this column, we find that all elements must be zero until we reach the first row, where

$$
u r_{1,1} a_{1,1}-a_{1,1} u c_{1,1}=0
$$

We know that $u r_{m, m}=u c_{m, m}$ for every $m$, so every possible $a_{1,1} \in \mathbb{C}$ satisfies this equation, meaning the element is indeterminate. We now consider the second column of the zero matrix on the rhs of Eq. (C11), starting with element $(N, 2)$ :

$$
u r_{N, N} a_{N, 2}-\left(a_{N, 1} u c_{1,2}+a_{N, 2} u c_{2,2}\right)=0 .
$$

We know $a_{N, 1}=0$, so we can write

$$
u r_{N, N} a_{N, 2}-a_{N, 2} u c_{2,2}=0
$$

and therefore $a_{N, 2}=0$. Next, we consider element $(N-1,2)$ :

$$
\begin{aligned}
& u r_{N-1, N-1} a_{N-1,2}+u r_{N-1, N} a_{N, 2} \\
& \quad-\left(a_{N-1,1} u c_{1,2}+a_{N-1,2} u c_{2,2}\right)=0 .
\end{aligned}
$$

Using previously known elements, we find that

$$
u r_{N-1, N-1} a_{N-1,2}-a_{N-1,2} u c_{2,2}=0 .
$$

Therefore, $a_{N-1,2}=0$. This continues up column 2 of the zero matrix until we get

$$
u r_{2,2} a_{2,2}-a_{2,2} u c_{2,2}=0
$$

Note that $a_{2,2}$ can be anything since $u r_{2,2}=u c_{2,2}$. Now, we consider element $(1,2)$ :

$$
\begin{aligned}
u r_{1,1} a_{1,2}+u r_{1,2} a_{2,2}-\left(a_{1,1} u c_{1,2}+a_{1,2} u c_{2,2}\right) & =0 \\
\left(u r_{1,1}-u c_{2,2}\right) a_{1,2}+u r_{1,2} a_{2,2}-a_{1,1} u c_{1,2} & =0 .
\end{aligned}
$$

By repeatedly applying this logic to each column of the zero matrix on the rhs of Eq. (C11), we conclude that the 
matrix $\mathbf{A}^{\prime}$ must have an upper triangular form. It then follows that element $(3,3)$ gives

$$
u r_{3,3} a_{3,3}-a_{3,3} u c_{3,3}=0
$$

and element $(2,3)$ gives

$$
\begin{aligned}
& u r_{2,1} a_{1,3}+u r_{2,2} a_{2,3}+u r_{2,3} a_{3,3} \\
& \quad-\left(a_{2,1} u c_{1,3}+a_{2,2} u c_{2,3}+a_{2,3} u c_{3,3}\right)=0, \\
& u r_{2,1} a_{1,3}+\left(u r_{2,2}-u c_{3,3}\right) a_{2,3}+u r_{2,3} a_{3,3}-a_{2,2} u c_{2,3}=0 .
\end{aligned}
$$

Considering element $(1,3)$ gives

$$
\begin{aligned}
& u r_{1,1} a_{1,3}+u r_{1,2} a_{2,3}+u r_{1,3} a_{3,3} \\
& \quad-\left(a_{1,1} u c_{1,3}+a_{1,2} u c_{2,3}+a_{1,3} u c_{3,3}\right)=0 \\
& \left(u r_{1,1}-u c_{3,3}\right) a_{1,3}+u r_{1,2} a_{2,3}+u r_{1,3} a_{3,3}-a_{1,1} u c_{1,3} \\
& \quad-a_{1,2} u c_{2,3}=0 .
\end{aligned}
$$

Therefore, these elements are all functions of elements that have already been computed when previously solving columns of the zero matrix on the righthand side of Eq. (C11) and the indeterminate diagonal elements of $\mathbf{A}^{\prime}$. By repeated application of this process, we can generate an equation for each upper triangular element, $(P, Q)$ with $P<Q$, of the zero matrix on the righthand side of Eq. (C11) as

$$
\left(u r_{P, P}-u c_{Q, Q}\right) a_{P, Q}+\sum_{q=P+1}^{Q} u r_{P, q} a_{q, Q}-\sum_{s=P}^{Q-1} u c_{s, Q} a_{P, s}=0 .
$$

Rearranging, we can write

$$
\begin{aligned}
& {\left[-\sum_{s=P}^{Q-1} u c_{s, Q} a_{P, s}\right]+\left(u r_{P, P}-u c_{Q, Q}\right) a_{P, Q}} \\
& \quad+\sum_{q=P+1}^{Q} u r_{P, q} a_{q, Q}=0, \\
& {\left[u r_{P, Q} a_{Q, Q}-\sum_{s=P}^{Q-1} u c_{s, Q} a_{P, S}\right]+\left(u r_{P, P}-u c_{Q, Q}\right) a_{P, Q}} \\
& \quad+\sum_{q=P+1}^{Q-1} u r_{P, q} a_{q, Q}=0 .
\end{aligned}
$$

In summary, we find that the diagonal elements, $a_{m, m}$ with $m=1, \ldots, N$, are indeterminate but that all other elements in the matrix $\mathbf{A}^{\prime}$ can be computed from these diagonals by solving a series of linear equations.

\section{APPENDIX D: SELECTING OPTIMAL WAVELENGTHS}

For a given reflector stack of the architecture presented in Sec. III A, we must determine the optical center-wavelengths of the three filters, the three optimal laser wavelengths for RM characterization, and the optimal laser wavelength for imaging. First, we approximate each longpass filter transmission function with a sigmoid function, $f$ :

$$
f\left(\lambda, \lambda_{\text {filt }}\right)=\frac{\tau_{\max }}{1+e^{-\frac{\alpha}{\tau_{\max }}\left(\lambda-\lambda_{\text {filt }}\right)}},
$$

where $\tau_{\max }$ is the maximum power transmission (fixed here as 0.8 based on real component data), $\alpha$ determines the steepness of the filter (measured to be about 0.73 based on numerous commercially available filters, e.g., ThorLabs FELH0850, which represents an approximately 5-nm wavelength change for $10 \%-90 \%$ normalized transmission), and $\lambda_{\text {filt }}$ is the center wavelength of the filter. Consider three laser wavelengths for RM characterization $\left(\lambda_{1}<\lambda_{2}<\lambda_{3}\right)$. We define a matrix $\mathbf{F}$ containing different values of the filter transmission function $f$ :

$$
\mathbf{F}=\left(\begin{array}{lll}
f\left(\lambda_{1}, \lambda_{a}\right) & f\left(\lambda_{1}, \lambda_{b}\right) & f\left(\lambda_{1}, \lambda_{c}\right) \\
f\left(\lambda_{2}, \lambda_{a}\right) & f\left(\lambda_{2}, \lambda_{b}\right) & f\left(\lambda_{2}, \lambda_{c}\right) \\
f\left(\lambda_{3}, \lambda_{a}\right) & f\left(\lambda_{3}, \lambda_{b}\right) & f\left(\lambda_{3}, \lambda_{c}\right)
\end{array}\right),
$$

where $\lambda_{a}<\lambda_{b}<\lambda_{c}$ are the center wavelengths of the three filters with transmission curves described by Eq. (D1). We can maximize the difference between the filters' behavior at the interrogation wavelengths by minimizing the condition number of $\mathbf{F}, \kappa(\mathbf{F})$, which gives our objective function $g$ :

$$
g\left(\lambda_{1}, \lambda_{2}, \lambda_{3}, \lambda_{a}, \lambda_{b}, \lambda_{c}\right)=\kappa(\mathbf{F})=\left\|\mathbf{F}^{-1}\right\|\|\mathbf{F}\|,
$$

where $\|\ldots\|$ is a matrix norm. We also want to ensure that, at the imaging wavelength $\lambda_{4}\left(>\lambda_{3}\right)$, the reflector stack transmits significantly more light than at the longest characterization wavelength $\lambda_{3}$. This is essential for efficient illumination and imaging. To quantify this case, we define a ratio $\beta$ of the transmittances of the final filter in the stack at wavelengths $\lambda_{3}$ and $\lambda_{4}$ as

$$
\beta=\frac{f\left(\lambda_{4}, \lambda_{c}\right)}{f\left(\lambda_{3}, \lambda_{c}\right)} .
$$

To account for the limitations of a tunable laser source, we must also constrain the maximum and minimum laser characterization wavelengths as well as the spacing between wavelengths. Finally, we must limit the difference between the maximum and minimum laser characterization wavelengths to be less than the fiber spectral bandwidth so that the first-order model remains valid. The full optimization problem is then 


$$
\begin{aligned}
& \min _{\lambda_{1}, \lambda_{2}, \lambda_{3}, \lambda_{a}, \lambda_{b}, \lambda_{c}} g\left(\lambda_{1}, \lambda_{2}, \lambda_{3}, \lambda_{a}, \lambda_{b}, \lambda_{c}\right) \\
& \text { s.t. } \lambda_{\min }<\lambda_{1}<\lambda_{2}<\lambda_{3}<\lambda_{4}<\lambda_{\max }, \\
& \lambda_{2}-\lambda_{1}, \lambda_{3}-\lambda_{2}, \lambda_{4}-\lambda_{3}>\Delta \lambda_{\mathrm{las}}, \\
& \lambda_{4}-\lambda_{1}<\Delta \lambda_{\mathrm{fib}}, \\
& \lambda_{\min }<\lambda_{a}<\lambda_{b}<\lambda_{c}<\lambda_{\max }, \\
& \beta>\beta_{\min },
\end{aligned}
$$

where $\lambda_{\min }$ and $\lambda_{\max }$ are the minimum and maximum range of the tunable laser, $\Delta \lambda_{\text {las }}$ is the minimum tuning step of the laser, and $\Delta \lambda_{\text {fib }}$ is the spectral bandwidth of the fiber.

Here, we set $\lambda_{\min }$ and $\lambda_{\max }$ to near-infrared wavelengths of $840 \mathrm{~nm}$ and $860 \mathrm{~nm}$, respectively [4]. We set $\Delta \lambda_{\text {las }}$ to $0.2 \mathrm{~nm}$ to reflect a typical tuning step of commercial tunable lasers. Note that $\Delta \lambda_{\text {fib }}$ is set to $7 \mathrm{~nm}$, representing a reasonable spectral bandwidth for a fiber of length about $2 \mathrm{~m}$ [32,33]. Finally, we choose $\beta_{\min }=10$ to provide acceptable isolation between characterization and imaging. With the objective and constraints defined, optimization is performed using a genetic algorithm.

\section{APPENDIX E: SIMULATING REFLECTORS}

We use two approaches for simulating reflectors. The first, used for simulated TMs, is to directly generate random reflector PMs. To do this, we first create a diagonal matrix with integers $1, \ldots, 2 M$ along the main diagonal in some permutation. Distinct permutations are used for the main diagonals of the three separate reflectors. Random permutations of the integers $1, \ldots, 2 M-1$ are then used to create the subdiagonals and super-diagonals of these reflector matrices, mimicking the mode coupling that would be expected in real reflectors. For the resultant matrix $\mathbf{R}^{\prime}$, the singular-value decomposition is computed as

$$
\mathbf{R}^{\prime}=\mathbf{U}_{R} \mathbf{S}_{R} \mathbf{V}_{R}^{H},
$$

where $\mathbf{U}_{R}$ and $\mathbf{V}_{R}$ are unitary, $\mathbf{S}_{R}$ is a diagonal matrix containing singular values of $\mathbf{R}$, and $(\ldots)^{H}$ represents a Hermitian transpose. The matrix is then reconstructed from the left- and right-singular vector matrices $\left(\mathbf{U}_{R}\right.$ and $\mathbf{V}_{R}$, respectively) but with the singular values (diagonals of $\mathbf{S}_{R}$ ) replaced by a new set, $s_{m}=m / 2 M$ with $m=1, \ldots, 2 M$. This ensures that the requirement for distinct eigenvalues (see Sec. II B) is satisfied.

The second approach, used for experimentally measured TMs, is a physically realistic simulation of reflector stacks like that shown in Fig. 3(a). This approach is achieved by extending the propagation matrix method used for simulating multilayer optical materials (Bragg reflectors or stacks) [47]. The reflector is modeled as a series of layers, as shown in Fig. 3(a), and the propagation operator used is
2D Fresnel propagation, applied separately to each polarization. This method is in contrast to the conventional propagation matrix method in which the propagation operator is the complex exponential propagation operator $e^{-i k z}$, where $z$ is the distance and $k$ is the wave number. The Fresnel propagation operator enables computation of output fields $\mathbf{p}_{H}^{\prime}$ and $\mathbf{p}_{V}^{\prime}$ (where subscripts $H$ and $V$ denote horizontal and vertical polarizations, respectively), resulting from input fields $\mathbf{p}_{H}$ and $\mathbf{p}_{V}$ propagating a distance $l$ through a layer as

$$
\mathbf{p}_{H}^{\prime}=\mathcal{F}^{-1}\left(\mathcal{F}\left(\mathbf{p}_{H}\right) e^{-i\left[\left(2 \pi^{2}\right) / k\right]\left(\xi_{x}^{2}+\xi_{y}^{2}\right) l}\right),
$$

where $\mathcal{F}$ is the discrete Fourier transform, $n$ is the refractive index of the layer, $\lambda$ is the wavelength, $k=[(2 \pi n) / \lambda], \xi_{x}$ and $\xi_{y}$ are coordinates in the Fourier plane, and constant factors are neglected [48]. This method is repeated for $\mathbf{p}_{V}$, giving a $2 \mathrm{D}$ complex vector at each point.

Using this modified method, the reflector stack is simulated using three types of layers: pure glass, glass with a wire-grid polarizer metasurface on the top, and optical filters. Here, we simulate absorptive optical filters, but reflective filters would work equally well. The filters have transmission curves typical of commercially available components, and the center wavelengths are determined using the process in Appendix D.

The stack simulated here comprises a 1-mm-thick layer of glass, followed by the three filters in succession, each $3 \mathrm{~mm}$ thick. The metasurfaces, negligibly thin for propagation purposes, are placed between the glass and the first filter, between the first and second filters, and between the second and third filters. The glass and filters have matched refractive indices of 1.52, and for simplicity, we neglect the imaginary part of the refractive index, i.e., distancedependent loss. Therefore, the only internal reflections occur at the metasurfaces, reflective filters (if used), and the final glass-air interface. Each metasurface is simulated by randomly generating diattenuating partial-polarizer Jones matrices for each sampled point of the field. Each point is assigned a diattenuation angle drawn from a uniform distribution, $\theta_{D} \sim U(-\pi, \pi)$, and a diattenuation drawn from a uniform distribution, $D \sim U(0,1)$. The transmitted light at each pixel is computed by multiplying the sampled field vector by the relevant Jones matrix:

$$
\begin{aligned}
\left(\begin{array}{c}
p_{t H} \\
p_{t V}
\end{array}\right)= & \sqrt{\alpha}\left(\begin{array}{cc}
\cos \theta_{D} & -\sin \theta_{D} \\
\sin \theta_{D} & \cos \theta_{D}
\end{array}\right)\left(\begin{array}{cc}
1 & 0 \\
0 & \sqrt{\frac{1-D}{1+D}}
\end{array}\right) \\
& \times\left(\begin{array}{cc}
\cos \theta_{D} & \sin \theta_{D} \\
-\sin \theta_{D} & \cos \theta_{D}
\end{array}\right)\left(\begin{array}{l}
p_{H}^{\prime} \\
p_{V}^{\prime}
\end{array}\right) .
\end{aligned}
$$

The light reflected is then given by 


$$
\begin{aligned}
\left(\begin{array}{c}
p_{r H} \\
p_{r V}
\end{array}\right)= & \sqrt{\alpha}\left(\begin{array}{cc}
\cos \theta_{D} & -\sin \theta_{D} \\
\sin \theta_{D} & \cos \theta_{D}
\end{array}\right)\left(\begin{array}{cc}
0 & 0 \\
0 & 1-\sqrt{\frac{1-D}{1+D}}
\end{array}\right) \\
& \times\left(\begin{array}{cc}
\cos \theta_{D} & \sin \theta_{D} \\
-\sin \theta_{D} & \cos \theta_{D}
\end{array}\right)\left(\begin{array}{l}
p_{H}^{\prime} \\
p_{V}^{\prime}
\end{array}\right),
\end{aligned}
$$

where $\alpha$ is a parameter representing overall power loss in the metasurface due to plasmonic losses, set to a typical value of 0.8 here [17].

The next step is to determine the overall PMs at the imaging and characterization wavelengths, $\mathbf{R}_{\lambda_{q}}, q=1, \ldots, 4$, and the $\mathrm{TM}$ of the reflector at the imaging wavelength, $\mathbf{A}_{\text {refl }}$. This step is achieved by iteratively propagating different input fields forwards and backwards through the stack until a steady-state solution is reached. This process is repeated at each of the desired wavelengths.

The input fields chosen may be derived from the columns of the fiber TM (the modes of the fiber) because any field exiting the fiber must be a linear combination thereof. However, the modes of the fiber may be expressed in some particular basis, e.g., Laguerre-Gauss modes for MMF, and must first be converted to the Cartesian basis so that Fresnel propagation can be applied. The light coupled back into the fiber can only be coupled into a linear combination of the reverse-propagating (i.e., complex conjugated) fiber modes. Therefore, the steady-state reflected field is reexpressed in the fiber mode basis. This means the reflector matrices have the same size as the fiber TM $\left(\in \mathbb{C}^{2 M \times 2 M}\right)$, but $\mathbf{A}_{\text {refl }}$ will, in general, be $\in \mathbb{C}^{2 N \times 2 M}$. Because the $2 N$-dimensional vector output of $\mathbf{A}_{\text {refl }}$ is in a Cartesian pixel basis, it is straightforward to compute sample illumination as per Sec. II E.

\section{APPENDIX F: SIMULATING FIBER TMS}

The simulated fiber TMs described in Sec. III B are generated using a model comprising a MCF with large core-to-core coupling. The 16 spatial pixels are considered to represent light-guiding cores organized in a rectangular $4 \times 4$ grid. The power coupling between a given core at the input and a given core at the output is modeled as decreasing exponentially with the squared lateral distance (arbitrary units) between them, i.e.,

$$
p_{\alpha, \beta}=e^{\left\{-\left[\left(x_{\alpha}-x_{\beta}\right)^{2}+\left(y_{\alpha}-y_{\beta}\right)^{2}\right] /\left(\sigma_{\mathrm{fib}}^{2}\right)\right\}},
$$

where $p_{\alpha, \beta}$ is the power coupled between core $\alpha$ at the input [with coordinates $\left(x_{\alpha}, y_{\alpha}\right)$ ] and core $\beta$ at the output [with coordinates $\left.\left(x_{\beta}, y_{\beta}\right)\right]$. Here, $\sigma_{\text {fib }}^{2}$ is a coupling parameter, which we set to be 3 . This model is therefore a Gaussian power-coupling model, which reflects empirical observations of real MCFs [29]. The power-coupling profile is duplicated for the second polarization. The complex phase of each element is drawn randomly from a uniform distribution, $\theta_{\alpha, \beta, s_{\text {in }}, s_{\text {out }}} \sim U(-\pi, \pi)$, where $s_{\text {in }}=1,2$ and $s_{\text {out }}=1,2$ indicate the input and output polarizations, respectively. This method gives a combined expression for each matrix element:

$$
a_{\alpha, \beta, s_{\text {in }}, s_{\text {out }}}=e^{\left\{\left[\left(x_{\alpha}-x_{\beta}\right)^{2}+\left(y_{\alpha}-y_{\beta}\right)^{2}\right] /\left(\sigma_{\text {fib }}^{2}\right)\right\}} e^{i \theta_{\alpha, \beta, s_{\text {in }}, s_{\text {out }}}} .
$$

The simulated TM, A, is formed of all elements $a_{\alpha, \beta, s_{\mathrm{in}}, s_{\mathrm{out}}}$ in some ordering such that each pair $\left(\beta, s_{\text {in }}\right)$ defines a unique row index and each pair $\left(\alpha, s_{\text {out }}\right)$ defines a unique column index. Then, $\mathbf{A}$ is decomposed using singular-value decomposition, and to ensure $\mathbf{A}$ is nonunitary but still invertible, new singular values, $s_{n}, n=1, \ldots, 32$, are generated such that $s_{n}=n / 32, n=1, \ldots, 32$. If using the zeroth-order model, the simulated matrix $\mathbf{A}$ is used as the TM at all wavelengths. If using the first-order model, the simulated matrix is used as the TM at wavelength $\lambda_{1}, \mathbf{A}_{\lambda_{1}}$, and the simulated TMs at the other wavelengths are generated from the matrix logarithm of $\mathbf{A}_{\lambda_{1}}$ [d $\mathbf{A}$ from Eq. (10)]; we then apply Eq. (7) to obtain $\mathbf{A}_{\lambda_{2}}$, $\mathbf{A}_{\lambda_{3}}, \mathbf{A}_{\lambda_{4}}$.

[1] T. Cižmár and K. Dholakia, Exploiting Multimode Waveguides for Pure Fibre-Based Imaging, Nat. Commun. 3, 1027 (2012).

[2] S. A. Vasquez-Lopez, R. Turcotte, V. Koren, M. Plöschner, Z. Padamsey, M. J. Booth, T. Čižmár, and N. J. Emptage, Subcellular Spatial Resolution Achieved for Deep-Brain Imaging In Vivo Using a Minimally Invasive Multimode Fiber, Light Sci. Appl. 7, 110 (2018).

[3] M. Gataric, G. S. D. Gordon, F. Renna, A. G. C. P. Ramos, M.P. Alcolea, and S.E. Bohndiek, Reconstruction of Optical Vector-Fields with Applications in Endoscopic Imaging, IEEE Trans. Med. Imaging 38, 955 (2019).

[4] G. S. D. Gordon, J. Joseph, T. Sawyer, A. J. Macfaden, C. Williams, T. D. Wilkinson, and S. E. Bohndiek, Full-Field Quantitative Phase and Polarisation-Resolved Imaging through an Optical Fibre Bundle, Opt. Express 27, 23929 (2019).

[5] D. Loterie, S. Farahi, I. Papadopoulos, A. Goy, D. Psaltis, and C. Moser, Digital Confocal Microscopy through a Multimode Fiber, Opt. Express 23, 23845 (2015).

[6] S. Rotter and S. Gigan, Light Fields in Complex Media: Mesoscopic Scattering Meets Wave Control, Rev. Mod. Phys. 89, 015005 (2017).

[7] G. J. Dunning and R. C. Lind, Demonstration of Image Transmission through Fibers by Optical Phase Conjugation, Opt. Lett. 7, 558 (1982).

[8] R. Di Leonardo and S. Bianchi, Hologram Transmission through Multi-mode Optical Fibers, Opt. Express 19, 247 (2011).

[9] S. Farahi, D. Ziegler, I. N. Papadopoulos, D. Psaltis, and C. Moser, Dynamic Bending Compensation While Focusing through a Multimode Fiber, Opt. Express 21, 22504 (2013).

[10] M. Plöschner, T. Tyc, and T. Čižmár, Seeing through Chaos in Multimode Fibres, Nat. Photonics 9, 529 (2015).

[11] H. Chen, N. K. Fontaine, R. Ryf, D. T. Neilson, P. Winzer, S. Gross, N. Riesen, M. J. Withford, J. Li, J. C. Alvarado, S. Wittek, J. Du, Z. He, J. E. A. Lopez, and R. AmezcuaCorrea, Remote Mode-Forming over Multimode Fiber 
Employing Single-Ended Channel Estimation, in 2018 European Conference on Optical Communication (ECOC), Rome (IEEE, 2018), Vol. 1, pp. 1-3.

[12] R. Y. Gu, R. N. Mahalati, and J. M. Kahn, Design of Flexible Multi-mode Fiber Endoscope, Opt. Express 23, 26905 (2015).

[13] S. Y. Lee, B. E. Bouma, and M. Villiger, Reciprocity in Measuring Multimode Fiber Transmission, in 2018 IEEE Photonics Conference (IPC), Reston, VA (IEEE, 2018), pp. 1-2.

[14] R. Y. Gu, Rigid and Flexible Multimode Fiber Endoscopes, Ph.D. thesis, Stanford University, 2017.

[15] J. Carpenter, B. J. Eggleton, and J. Schröder, $110 \times 110$ Optical Mode Transfer Matrix Inversion, Opt. Express 22, 96 (2014).

[16] G. Gordon, M. Crisp, R. Penty, T. Wilkinson, and I. White, Feasibility Demonstration of a Mode-Division Multiplexed MIMO-Enabled Radio-over-Fiber Distributed Antenna System, J. Lightwave Technol. 32, 3512 (2014).

[17] C. Williams, R. Bartholomew, G. Rughoobur, G. S. D. Gordon, A. J. Flewitt, and T. D. Wilkinson, Fabrication of Nanostructured Transmissive Optical Devices on ITO-Glass with UV1116 Photoresist Using High-Energy Electron Beam Lithography, Nanotechnology 27, 485301 (2016).

[18] Z. Zhang, F. Dong, T. Cheng, K. Qiu, Q. Zhang, W. Chu, and X. Wu, Nano-fabricated Pixelated Micropolarizer Array for Visible Imaging Polarimetry, Rev. Sci. Instrum. 85, 105002 (2014).

[19] J. Vizet, S. Manhas, J. Tran, P. Validire, A. Benali, E. GarciaCaurel, A. Pierangelo, A. De Martino, and D. Pagnoux, Optical Fiber-Based Full Mueller Polarimeter for Endoscopic Imaging Using a Two-Wavelength Simultaneous Measurement Method, J. Biomed. Opt. 21, 071106 (2016).

[20] S. Fan and J. M. Kahn, Principal Modes in Multimode Waveguides, Opt. Lett. 30, 135 (2005).

[21] A. Yariv, Coupled-Mode Theory for Guided-Wave Optics, IEEE J. Quantum Electron. 9, 919 (1973).

[22] W. P. Huang, Coupled-Mode Theory for Optical Waveguides: An Overview, J. Opt. Soc. Am. A 11, 963 (1994).

[23] B. Redding, S. M. Popoff, and H. Cao, All-Fiber Spectrometer Based on Speckle Pattern Reconstruction, Opt. Express 21, 6584 (2013).

[24] D. Loterie, D. Psaltis, and C. Moser, Bend Translation in Multimode Fiber Imaging, Opt. Express 25, 6263 (2017).

[25] D. N. Payne and W. A. Gambling, Zero Material Dispersion in Optical Fibre, Electron. Lett. 11, 176 (1975).

[26] J. Carpenter, A Matlab File Containing the Optical Transfer Function of a Multimode Optical Fiber, https://doi.org/ 10.14264/uql.2016.678 (2016).

[27] D. P. Kingma and J. Ba, Adam: A Method for Stochastic Optimization (2014).

[28] Y. Yamamoto, AM and FM Quantum Noise in Semiconductor Lasers-Part I: Theoretical Analysis, IEEE J. Quantum Electron. 19, 34 (1983).

[29] G. S. D. Gordon, R. Mouthaan, T. D. Wilkinson, and S. E. Bohndiek, Coherent Imaging through Multicore Fibres with Applications in Endoscopy, J. Lightwave Technol. 1 (2019).

[30] Y. Choi, C. Yoon, M. Kim, T. D. Yang, C. Fang-Yen, R. R. Dasari, K. J. Lee, and W. Choi, Scanner-Free and WideField Endoscopic Imaging by Using a Single Multimode Optical Fiber, Phys. Rev. Lett. 109, 203901 (2012).
[31] S. M. Popoff, G. Lerosey, M. Fink, C. Boccara, and S. Gigan, Controlling Light through Optical Disordered Media: Transmission Matrix Approach, New J. Phys. 13, 123021 (2011).

[32] A. J. Thompson, Developing Endoscopic Instrumentation and Techniques for In Vivo Fluorescence Lifetime Imaging and Spectroscopy, Ph.D. thesis, Imperial College London, 2012.

[33] J. Carpenter, B. J. Eggleton, and J. Schröder, Observation of Eisenbud-Wigner-Smith States as Principal Modes in Multimode Fibre, Nat. Photonics 9, 751 (2015).

[34] B. Rahmani, D. Loterie, G. Konstantinou, D. Psaltis, and C. Moser, Multimode Optical Fiber Transmission with a Deep Learning Network, Light Sci. Appl. 7, 69 (2018).

[35] M. Yang, Z.-h. Liu, Z.-d. Cheng, J.-s. Xu, C.-f. Li, and G.-c. Guo, Deep Hybrid Scattering Image Learning, J. Phys. D 52, 115105 (2018).

[36] S. Turtaev, I. T. Leite, K. J. Mitchell, M. J. Padgett, D. B. Phillips, and T. Čižmár, Exploiting Digital Micromirror Device for Holographic Micro-endoscopy, in Emerging Digital Micromirror Device Based Systems and Applications XI, San Francisco, CA, edited by M. R. Douglass, B. L. Lee, and J. Ehmke (SPIE, 2019), Vol. 1093203, p. 2.

[37] M. Jang, H. Ruan, I. M. Vellekoop, B. Judkewitz, E. Chung, and C. Yang, Relation between Speckle Decorrelation and Optical Phase Conjugation (OPC)-Based Turbidity Suppression through Dynamic Scattering Media: A Study on In Vivo Mouse Skin, Biomed. Opt. Express 6, 72 (2015).

[38] T. Čižmár, M. Mazilu, and K. Dholakia, In Situ Wavefront Correction and Its Application to Micromanipulation, Nat. Photonics 4, 388 (2010).

[39] C. Williams, G. Rughoobur, A. J. Flewitt, and T. D. Wilkinson, Nanostructured Plasmonic Metapixels, Sci. Rep. 7, 7745 (2017).

[40] Polarsens: Sony' Polarization Image Sensor, https://www .sony-semicon.co.jp/products_en/IS/sensor5/index.html.

[41] A. P. Mosk, A. Lagendijk, G. Lerosey, and M. Fink, Controlling Waves in Space and Time for Imaging and Focusing in Complex Media, Nat. Photonics 6, 283 (2012).

[42] R. Bartels and G. W. Stewart, Algorithm 432: Solution of the Matrix Equation $A X+X B=C$ [F4], Commun. ACM 15, 820 (1972).

[43] R. A. Horn and C. R. Johnson, Matrix Analysis (Cambridge University Press, New York, 2013), p. 607.

[44] V. A. Marčenko and L. A. Pastur, Distribution of Eigenvalues for Some Sets of Random Matrices, Math. USSRSbornik 1, 457 (1967).

[45] S. M. Popoff, G. Lerosey, R. Carminati, M. Fink, A. C. Boccara, and S. Gigan, Measuring the Transmission Matrix in Optics: An Approach to the Study and Control of Light Propagation in Disordered Media, Phys. Rev. Lett. 104, 100601 (2010).

[46] E. Bodewig, Matrix Calculus (North Holland, Amsterdam, 1959).

[47] S. J. Orfanidis, Electromagnetic Waves and Antennas (Rutgers University, Piscataway, NJ, 2016), pp. 216-223.

[48] J. W. Goodman, Introduction to Fourier Optics, 2nd ed., McGraw-Hill Physical and Quantum Electronics Series (McGraw-Hill, New York, 1996).

[49] https://doi.org/10.17863/CAM.45121 